\title{
Regularite globale des solutions de systemes Elliptiques non lineaires
}

\author{
J.-P. RAYMOND
}

Abstract. We consider the regularity of functions $u \in W_{0}^{1, p}\left(\Omega, \mathbb{R}^{m}\right)$ solving elliptic degenerated or singular systems of the type:

$$
-\frac{\partial}{\partial x_{a}}\left(a_{i}^{a}(x, \nabla u(x))\right)=f^{\prime}(x, u(x))
$$

We suppose that $a, 5, v)$ satisfies a growth condition of order $(p-1)$ with respect to $v$.

We show that, if $p \leqslant 2$, the solutions of the system belong to $W^{2, n p /(n+p-2)}$, if $p>2$ and $n>2$, they belong to $W^{1, n /(n-2)}$ and if $p>2$ and $n=2$, they belong to $W^{1, r}$ for every $r<+\infty$.

Résumé: On s'intéresse à la régularité dans les espaces de Sobolev des fonctions $u$ de $W_{0}^{\prime, p}\left(\Omega, \mathbb{R}^{m}\right)$ solutions de systèmes elliptiques dégénérés ou singuliers du type:

$$
-\frac{\partial}{\partial x_{\mathrm{a}}}\left(a_{i}^{a}(x, \nabla u(x))\right)=f^{i}(x, u(x))
$$

On suppose que $a_{\mathrm{i}}^{\alpha}(x, v)$ a une croissance d'ordre $p-1$ en $v$.

On montre que si $p \leqslant 2$, les solutions du système appartiennent à $W^{2, n p /(n+p-2)}\left(\Omega, \mathbb{R}^{m}\right)$, si $p>2$ et $n>2$, ces solutions appartiennent à $W^{1, n p /(n-2)}$ et si $p>2$ et $n=2$, elles appartiennent à $W^{l, r}$ pour tout $r<+\infty$.

\section{INTRODUCTION}

On s'intéresse dans cet article à la régularité dans les espaces de Sobolev des fonctions $u$ de $W_{o}^{1, p}\left(\Omega, \mathbb{R}^{m}\right)$ solutions de systèmes elliptiques du type:

$$
-\frac{\partial}{\partial \overline{x_{\mathrm{a}}}}\left(a_{i}^{a}(x, \nabla u(x))\right)=f^{\prime}(x, u(x)) \text { dans } \mathrm{D}^{\prime}(\Omega)
$$

1980 Mathematics Subject Classification (1985 revision): 35J70, 35B65

Editorial de la Universidad Complutense. Madrid, 1989. 
avec $1 \leqslant i \leqslant m, \Omega$ étant un ouvert borné de $\mathbb{R}^{n}$. On s'intéresse plus précisément aux systèmes vérifiant la condition d'ellipticité suivante:

$$
\begin{aligned}
& a_{i j}^{\alpha \beta}\left(x, v^{\prime}\right) w_{a}^{j} w_{p}^{j} \geq\left. c\left|y^{p-2}\right| w\right|^{2}, \\
& \text { où } a_{j}^{\alpha \beta}(x, v)=\frac{\partial}{\partial \bar{w}_{\beta}^{j}} a_{i}^{\mathrm{a}}(x, v) .
\end{aligned}
$$

Lorsque $a_{j}^{\alpha}$ est de la forme $a_{r}^{\alpha}(x, b)=c(x, w) v_{a}^{i}$ des résultats de régularité dans les espaces $C^{\prime \mu \mu}$ ont été donněs dans [18], [19], [8] et pour des problèmes paraboliques associés à de tels systèmes dans [4]. Dans le cas scalaire $(m=1)$, on peut aussi consulter [3], [5], [17]. En l'absence de structure particulière pour $a_{1}^{a}$ on ne peut montrer que des résultats de régularité partielle de la forme:

Les solutions de (1.1) appartiennent à $C^{\prime \mu}\left(\Omega_{0}, \mathbb{R}^{m}\right)$, avec $\operatorname{Mes}\left(\Omega \backslash \Omega_{o}\right)=0$. (Cf. [7], [8]).

On peut par contre obtenir des résultats locaux ou globaux dans les espaces de Sobolev. Nous avons déjà étudié des systèmes du type (1.1) dans [10] et nous avons montré que, pour $1<p \leqslant 2$, les solutions de (1.1) appartiennent à $W_{10<}^{2 n p /(n+p-2)}\left(\Omega, \mathbb{R}^{m}\right)$, ce qui améliorait les résultats antérieurs ([9], [12], [13], [15], [16]). Nous nous proposons ici d'étudier la régularité jusqu'à la frontière. La méthode développée dans [10] consiste à montrer que l'on a:

$$
|\nabla u|^{[\mathrm{p}-2) / 2} \nabla^{2} u \in \mathrm{L}_{\text {lox }}^{2}\left(\Omega, \mathbb{R}^{\mathrm{m}}\right),
$$

puis, dans le cas $1<\mathrm{p} \leqslant 2$, à utiliser une inégalité de Hölder d'exposant négatif (lemme 2,1). On obtient alors à l'aide d'un processus itératif:

$$
u \in \mathrm{W}_{\mathrm{ioc}}^{2, \mathrm{p} /(\mathrm{n} * \mathrm{p}-2)}\left(\Omega, \mathbb{R}^{m}\right) \text {. }
$$

Dans le cas $p>2$, l'équation adjointe de $(1,1)$ vérifie une condition d'ellipticité du type (1.2), où $p$ est remplacé par $p^{\prime}=p /(p-1)$. Etant donné que l'on a $p^{\prime}<2$, la méthode précédente permet de montrer que les solutions de l'équation adjointe appartiennent à $W_{10 c}^{n, p^{\prime} /\left(n+p^{\prime}-2\right)}\left(\Omega, \mathbb{R}^{m} n\right)$. La régularité des solutions de (1.1) est alors déduite des relations de dualité (cf. [10]). A titre d'exemple, signalons que dans le cas particulier où l'on a:

$$
a_{i}^{\mathrm{a}}(x, v)=d(x)|v| \mathrm{p}-2, v_{\mathrm{a}}, \text { avec } 0<c_{1} \leqslant d(x) \leqslant c_{2},
$$

le système (1.1) s'écrit sous forme vectorielle:

$$
-\operatorname{div}\left(d(x)|\nabla u(x)|^{p-2} \nabla u(x)\right)=f(x, u(x))
$$

Une fonction $q$ de $L^{p}\left(\Omega, \mathbb{R}^{m}\right)$ sera une solution adjointe de (1.1) associée à une fonction $u$ de $W_{o}^{1, p}$ si elle vérifie: 


$$
\begin{gathered}
\operatorname{div}(q(x))=-f(x, u(x)) \text { dans } \mathrm{D}^{\prime}\left(\Omega, \mathbb{R}^{\mathrm{m}}\right) \text { et } \\
q(x)=d(x) \mid \nabla u(x)^{\mathrm{p}-2} \nabla u(x), \text { pour presque tout } x \text { de } \Omega .
\end{gathered}
$$

Nous détaillons jusqu'au paragraphe 3 les calculs relatifs à $p \leqslant 2$. Dans le paragraphe 4 , on introduit l'opérateur adjoint de $\left(a_{4}^{a}\right)$ et on établit les estimations correspondant à cet opérateur.

Au paragraphe 5, on indiquera comment développer pour l'équation adjointe de (1.1) dans le cas $p>2$ les techniques mises au point pour $p \leqslant 2$.

Nous démontrons que les solutions de (1.1) appartiennent à $W^{2, n p /(n+p-2)}\left(\Omega, \mathbb{R}^{m}\right)$ si $p \leqslant 2$ (théorème 3.2$)$, à $W^{1, n p /(n-2)}\left(\Omega, \mathbb{R}^{m}\right)$ si $p>2$ et si $n>2$ et à $W^{1 ، r}\left(\Omega, \mathbb{R}^{m}\right)$ pour tout $r<+\infty$ si $p>2$ et $n=2$ (théorème 5.4 ).

Ces résultats sont à notre connaissance nouveaux. Signalons aussi l'originalité de certaines techniques de démonstration:

-utilisation de l'inégalité de Hölder d'exposant négatif pour traiter la singularité à l'origine de $a_{i}^{a}(x,$.$) si p$ est inférieur à 2 ,

-utilisation de l'équation adjointe pour passer du cas dégénéré $(p>2)$ à un système avec singularité (le système adjoint présente une singularité à l'origine car on a $p^{\prime}<2$ ).

Ces deux techniques ont déjà été utilisées par l'auteur dans [10] pour l'étude de la régularité locale et interviennent ici pour l'estimation des dérivées du gradient des solutions de (1.1) dans les directions tangentes à la frontière de $\Omega$ pour $p \leqslant 2$ (proposition 3.3) el également pour l'estimation des dérivées dans les directions tangentes à la frontière des solutions adjointes de (1.1) pour $p \geqslant 2$ (proposition 5.5).

Notons enfin que les techniques classiques d'estimation des dérivées du gradient des solutions ou des solutions adjointes, dans la direction normale à la frontière de $\Omega$ ne sont pas applicables aux systèmes dégénérés ou singuliers. Ceci nous a conduit à développer une approche nouvelle de cette question (cf. les preuves des propositions 3.4 et 5.6).

\section{PRELIMINAIRES}

\section{Notations}

1. L'espace $W^{1, p}\left(\Omega, \mathbb{R}^{m}\right)$ désigne $\left[W^{1, p}(\Omega)\right]^{m}$. On adopte le même type de notation pour les autres espaces de fonctions vectorielles. 
2. Si $v$ est une fonction de $A \subset \mathbb{R}^{\mathrm{n}}$ dans $\mathbb{R}^{1},\|v\|_{\mathrm{s}}$ désigne la norme de $v$ dans $L^{\mathrm{s}}\left(\Omega, \mathbb{R}^{\prime}\right)$.

3. Les fonctions $a_{i}^{a}$ sont définies sur $\mathbb{R}^{\mathrm{n}} \times \mathbb{R}^{\mathrm{mn}}$, on pose

$$
a_{i \gamma}^{\mathrm{a}}(x, v)=\frac{\partial}{\partial x_{\gamma}} a_{i}^{\mathrm{a}}(x, v), \quad a_{i j}^{\mathrm{\alpha}}(x, v)=\frac{\partial}{\partial w_{\beta}^{\alpha}} a_{i}^{\mathrm{a}}(x, v)
$$

Les indices latins varient de 1 à $m$ et les indices grecs de 1 à $n$. Sauf mention contraire, on adopte la convention de sommation sur les indices répétés, (si plus de deux indices identiques apparaissent, la sommation opère par paire d'indices identiques entre un indice donné et le suivant le plus proche qui lui est identique).

4. Si $B$ désigne un vecteur ou une matrice, $|B|$ désigne la norme euclidienne de $B$.

5. Dans la suite, $\left(\mathrm{T}_{\alpha}^{\delta}\right)_{1 \leqslant \delta \leqslant n}$ désigne une matrice $n \times n, \delta$ désignant l'indice de ligne et $\alpha$ l'indice de colonne, la norme euclidienne de $\left(T_{a}^{b}\right)$ est notee $\left|T_{a}^{\delta}\right|$ et la norme euclidienne du vecteur ligne d'indice $\gamma$ de la matrice $\left(T_{\alpha}^{\delta}\right)$ est notée $\left[\mathrm{Tr}^{\mathrm{r}}\right.$.

6. Si $v$ est une fonction de $A \subset \mathbb{R}^{\mathrm{n}}$ dans $\mathbb{R}^{\prime}, \nabla v$ désigne le gradient de $v$ (il est à valeurs dans $\mathbb{R}^{\text {in }}$ ), $\nabla^{2} v$ le hessien de $v$. Les dérivées partielles premières sont notées $v_{\mathrm{a}}$ ( $\alpha$ pouvant être remplacé par n'importe quel indice grec) et les dérivées partielles secondes sont notées $v_{\mathrm{ap}}$.

7. On pose $\mathbb{R}_{+}^{n}=\left\{y \in \mathbb{R}^{\mathrm{n}} \mid y_{n} \geqslant 0\right\}$ et $\mathrm{B}(0, \rho)=\left\{y \in \mathbb{R}^{\mathrm{n}}|| y \mid \leqslant \rho\right\}$.

\section{Hypothèses}

$$
\Omega \text { est un ouvert borné de } \mathbb{R}^{n} \text { de classe } C^{2} \text {. }
$$

$$
\forall x \in \Omega, \forall u \in \mathbb{R}^{\mathrm{m}},|f(x, u)| \leqslant a_{1}|u|^{(\mathrm{ap}-1 /(\mathbf{n}-\mathrm{p})}+N(x), \text { avec } N \in L^{p}(\Omega) .
$$

Les fonctions $a_{i}^{a}$ appartienent à $C^{1}\left(\mathbb{R}^{\mathrm{n}} \times \mathbb{R}^{\mathrm{m} m} \backslash\left\{0_{\mathrm{m} n}\right\}\right)$

et vérifient la condition de symétrie $a_{i j}^{\alpha \beta} \equiv a_{j i}^{\beta a p}$ pour tout $i$, tout $j$, tout $\alpha$, tout $\beta$.

Il existe des constantes $a_{2}, \ldots a_{5}$ positives telles que, pour tout $x$ de $\Omega$, tout $v$ de $\mathbb{R}^{\mathrm{mn}} \backslash\left\{0_{\mathrm{mm}}\right\}$, tout $w$ de $\mathbb{R}^{\mathrm{mn}}$, on ait:

$$
\left|a_{i}^{\alpha}(x, v)\right| \leqslant a_{2}|v|^{\mathrm{p}-1}
$$




$$
\begin{aligned}
& \left|a_{i \gamma}^{u}(x, v)\right| \leqslant a_{3}|v|^{p-1}, \\
& \left|a_{i j}^{a p}(x, v)\right| \leqslant a_{4}|v|^{p-2}, \\
& a_{i j}^{\alpha}(x, v) \quad w_{a}^{\alpha} w_{\beta}^{j} \geqslant\left. a_{s}|v p-2| w\right|^{2} .
\end{aligned}
$$

Remarque: On aurait pu remplacer les constantes $a_{2}, a_{3}, a_{4}$ par des fonctions positives de $x$ appartenant à un espace $L^{\prime}(\Omega)$ convenablement choisi, en adoptant la même démarche, on aurait encore eu un résultat de régularité du type $u \in \mathrm{W}^{2, l}$, avec $/$ fonction de $r$ et $l<n p /(n+p-2)$.

\section{Lemmes préliminaires}

Lemme 2.1 (cf. [1] et [10]). Soient $0<r<1, r^{\prime}=r /(r-1)$ et $\Omega^{\prime}$ un ensemble mesturable de $\mathbb{R}^{n}$. Si f appartient à $L^{\prime}\left(\Omega^{\prime}\right)$ et si $\int_{\Omega^{\prime}}|g(x)|^{r} d x<+\infty$, on a:

$$
\left.\left\{\int_{\Omega} \mid f(x)\right)^{r} d x\right\}^{1 / t} \leqslant\left\{\int_{\Omega},|f(x) g(x)| d x \mid\left\{\int_{\Omega},|g(x)| r^{r} d x\right\}^{-1 / r}\right.
$$

Remarque: Ce lemme est une forme faible du théorème 2.6 de [1], l'hypothèse: $0<\int_{\Omega^{\prime}}|g(x)|^{r} d x$ n'est pas ici nécessaire (cf. [10], lemme 3.2).

Lemme 2.2: Si $p \leqslant 2$, il existe deux constantes $k_{1}>k_{2}>0$ telles que, pour tout $a \in \mathbb{R}^{\prime}$, pour tout $b \in \mathbb{R}^{\prime}$, on ait:

$$
k_{2} \leqslant \int_{0}^{1}|t a+(1-t) b|^{\mathrm{p}-2} d t(|a|+|b|)^{2-p} \leqslant k_{1} .
$$

Preuve: Montrons tout d'abord l'inégalité de droite. On a:

$$
|t a+(l-t) b| \geqslant|t| a|-(l-t)| b|| \text { et }|t a+(l-t) b|^{p-2} \leqslant|t| a|-(1-t)| b||^{p-2} \text {. }
$$

On en déduit:

$$
\begin{aligned}
I=\int_{0}^{1}|t a+(1-t) b|^{p-2} d t & \leqslant \int_{0}^{c}\left(-t(|a|+|b|)+|b|^{p-2} d t\right. \\
& +\int_{c}^{1}\left(t(|a|+|b|)-\left.|b|\right|^{p-2} d t\right.
\end{aligned}
$$


avec $c=\frac{|b|}{|a|+|b|}$. Par le calcul, on obtient:

$$
I \leqslant \frac{|b|^{p-1}+|a|^{p-1}}{(p-1)(|a|+|b|)},
$$

Compte tenu de $|a|^{p-1}+|b|^{p-1} \leqslant 2^{2-p}\left(|a|+\left.|b|\right|^{p-1}\right.$, on a:

$$
\int_{0}^{1}|t a+(1-t) b|^{p-2}(|a|+|b|)^{2-p} \leqslant \frac{2^{2-p}}{p+1} \text {. }
$$

L'inégalitê de gauche se montre par disjonction de cas.

ler cas. Supposons que l'on ait: $\frac{3}{4}|a| \leqslant|b| \leqslant \frac{5}{4}|a|$ ou $\frac{3}{4}|b| \leqslant|a| \leqslant$

$\frac{5}{4}|b|$. Ces deux sous-cas se traitant de manière identique, on suppose que l'on a:

$\frac{3}{4}|a| \leqslant|b| \leqslant \frac{5}{-4}|a|$. On a alors les inégalités suivantes:

$$
|t a+(1-t) b| \leqslant t|a|+(1-t)|b| \leqslant \frac{t}{4}|a|+\frac{5}{4}|a| .
$$

On en déduit:

$$
I \geqslant \int_{0}^{1}\left(\frac{t}{4}|a|+\frac{5}{4}|a|\right)^{p-2} d t=\left(\frac{|a|}{4}\right)^{p-2}\left(\frac{6^{p-1}-5^{p-1}}{p-1}\right)
$$

Comme de plus, on a: $\left(|a|+|b|^{2-p} \geqslant\left(\frac{7}{4}\right)^{2-p}|a|^{2-p}\right.$, on obtient:

$$
I(|a|+\mid b)^{2-p} \geqslant(7)^{2-p}\left(\frac{6^{p-1}-5^{p-1}}{\overline{p-T}}\right) \text {. }
$$

2éme cas. On a $|a| \notin\left[\frac{3}{4}|b|, \frac{5}{4}|b|\right]$ et $|b| \notin\left[\frac{3}{4}|a|, \frac{5}{-4}|a|\right]$, soit encore $|b|<\frac{3}{4}|a|$ ou $|a|<\frac{3}{4}|b|$. Plaçons nous dans le cas où: $|b| \leqslant$ $\frac{3}{4}|a|$. On a alors: 


$$
I \geqslant \int_{0}^{1}\left(t(|c|-|b|)+|b|^{p-2} \mathrm{dt}=\frac{|a|^{p-1}-|b|^{p-1}}{(p-1)(|a|-|b|)} \geqslant|a|^{p-2} \frac{1-\left(3 / 4^{p-1}\right.}{(p-1)}\right.
$$

D'où: $I(|a|+|b|)^{2-p} \geqslant \frac{1-(3 / 4)^{p-1}}{(p-1)}$.

\section{RESULTATS DE REGULARITE}

Commençons par rappeler le résultat suivant:

Proposition 3.1: Si $p \leqslant 2$ et si les hypothèses (2.1) à (2.7) sont vérifiées, les solutions de (1.l) appartiennent à $W_{i a x}^{\text {apAn+p-2) }}\left(\Omega, \mathbb{R}^{\mathrm{m}}\right)$.

Un résultat de ce type est démontré dans [10] lorsque les fonctions $a_{i}^{a}$ ne dépendent pas explicitement de $x$. Le résultat de la proposition 3.1 se démontre de manière identique, il suffit de tenir compte des termes où figure $a_{i \gamma}^{a}$ qui apparaissent dans les calculs et de majorer ces termes convenablement.

Nous démontrons dans ce paragraphe le résultat principal de cet article dans le cas $p \leqslant 2$.

Théorème 3.2: Si $p \leqslant 2$ et si les hypothèses (2.1) à (2.7) sont vérifiées, les solutions de (1.1) appartiennent à $\mathrm{W}^{2, \mathrm{np} / \mathrm{n}+\mathrm{p}-2)}\left(\Omega, \mathbb{R}^{\mathrm{m}}\right)$.

Pour établir le théorème 3.2 , il suffit de montrer que pour tout pont $x_{\mathrm{o}}$ de $\partial \Omega$ et pour un voisinage $V$ de ce point, les solutions de (1.1) appartiennent à $\mathrm{W}^{2, n p(n-p+2)}\left(\Omega \cap V, \mathbb{R}^{\mathrm{m}}\right)$. De façon à se ramener à un problème à frontière droite, on considère un difféomorphisme $\psi$ de classe $\mathrm{C}^{2}$ et deux voisinages $V$ et $V$ 'du point frontière $x_{0}$ vérifiant:

$$
\begin{gathered}
V \subset V^{\prime}, \\
\psi(V)=B(0, R), \psi(V)=B(0, r), 0<r<R, \\
\psi(\Omega \cap V)=\mathbb{R}_{+}^{\mathrm{n}} \cap B(0, r), \psi\left(\Omega \cap V^{\prime}\right)=\mathbb{R}_{+}^{\mathrm{n}} \cap B(0, R) .
\end{gathered}
$$

On pose $\psi=\left(\psi^{a}\right)_{1<a<n}$ et $\psi^{-1}=\left(\psi^{-1, a}\right)_{1 \leqslant a<n}$. On considère le système (1.1) sous la forme:

$$
\int_{\Omega} a_{i}^{a}(x, \nabla u(x)) \Phi_{a}^{i}(x) d x=\int_{\Omega} f(x, u(x)) \Phi(x) d x
$$

pour tout $\Phi$ de $W_{0}^{1 \cdot p}\left(\Omega, \mathbb{R}^{m}\right)$. 
On suppose maintenant que l'on a: $\Phi \in \mathrm{W}_{0}^{\mathrm{l} p}\left(\Omega \cap V^{\prime}, \mathbb{R}^{\mathrm{m}}\right)$. On effectue le changement de variable $y=\psi(x)$ dans (3.1). On pose:

$$
\begin{aligned}
& \tilde{u}(y)=u\left(\psi^{-1}(y)\right), \tilde{f}(y, u)=f\left(\psi^{-1}(y), u\right), \\
& \tilde{\Phi}(y)=\Phi\left(\psi^{-1}(y)\right), A_{i}^{a}(y, w)=a_{i}^{a}\left(\psi^{-1}(y), w\right), \\
& A_{r y}^{\mathrm{u}}(y, w)=\frac{\partial}{\partial y_{y}} A_{i}^{a}(y, w), \\
& A_{i j}^{\alpha \beta}(y, w)=a_{i j}^{\alpha \beta}\left(\psi^{-1}(y), w\right), \\
& J^{-1}(y)=\operatorname{det}\left[\left(\frac{\partial \psi^{-1 . a}}{\partial y_{\beta}}(y)\right)_{\substack{1<\alpha<n \\
1<\beta \leqslant n}}\right], \\
& J_{\gamma}^{-1}(y)=\frac{\partial}{\partial y_{\gamma}} J^{-1}(y), \\
& T^{\mathrm{b}}(y)=\nabla \psi^{\mathrm{s}}\left(\psi^{-1}(y)\right) \text { et } T_{\beta}^{\mathrm{s}}\left(\psi^{-1}(y)\right)=\psi_{\beta}^{s}\left(\psi^{-1}(y)\right) \text {. }
\end{aligned}
$$

Avec ces notations, on a:

$$
\begin{gathered}
u(x)=\check{u}(y), \quad \nabla u(x)=\check{u}_{\mathrm{a}}(y) T^{\mathrm{b}}(y), \\
\Phi(x)=\tilde{\Phi}(y), \tilde{\Phi} \in \mathbf{W}_{0}^{\llcorner\cdot p}\left(\mathbb{R}_{+}^{\mathrm{n}} \cap B(0, R), \mathbb{R}^{\mathrm{m}}\right), \\
\dot{\Phi}_{\mathrm{a}}(x)=\tilde{\Phi}_{b}(y) T_{\mathrm{a}}^{\mathrm{b}}(y),
\end{gathered}
$$

Par changement de variable, l'équation (3.1) devient:

$$
\begin{aligned}
& \int_{\mathbb{R}_{+}^{n}} J^{-1}(y) A_{i}^{\mathrm{a}}\left(y, T^{\mathrm{s}}(y) \tilde{u}_{6}(y)\right) T_{\mathrm{a}}^{\mathrm{b}}(y) \dot{\Phi}_{\delta}(y) d y \\
& =\int_{\mathbb{R}_{+}^{n}} J^{-1}(y) \tilde{f}(y, \tilde{u}(y)) \tilde{\Phi}^{\prime}(y) d y,
\end{aligned}
$$

pour tout $\tilde{\Phi} \in \mathbf{W}_{0}^{1, p}\left(\mathbb{R}_{+}^{n} \cap B(0, R), \mathbb{B}^{\mathrm{m}}\right)$.

Remarque: $\left(T_{\mathrm{a}}^{8}(y)\right)$ est une matrice inversible et on a:

$$
\left(T_{a}^{s}(y)\right)^{-1}=\psi_{b}^{1 \cdot a}(y) .
$$

Etant donné que $\Omega$ est de classe $C^{2}$, il est toujours possible de choisir $V^{\prime \prime}$ suf- 
fisamment petit et des constantes positives $b_{1}$ à $b_{5}$ tels que, pour tout $y$ de $B(0, R)$, tout $\gamma \in\{1, \ldots, n\}$, on ait:

$$
\begin{aligned}
& \forall \gamma \in\{1, \ldots, n\},\left[T^{3}(y)\right] \geqslant b_{1}>0, \\
& \left|T_{\mathrm{a}}^{\mathrm{b}}(y)\right| \leqslant b_{2}, \\
& \left|\left(T_{\mathrm{a}}^{\mathrm{s}}(y)\right)^{-1}\right| \leqslant b_{3}, \\
& 0<b_{4} \leqslant J^{-1}(y) \leqslant b_{s},
\end{aligned}
$$

où $[T]$ est défini au paragraphe 2 .

La démonstration du théorème 3.2 repose essentiellement sur les propositions suivantes.

Proposition 3.3: Si une solution ù de (3.3) appartient à $W^{l, s}\left(\mathbb{R}_{+}^{n} \cap B(0, r), \mathbb{R}^{\mathrm{m}}\right)$ avec $p \leqslant 2$ et $p \leqslant s \leqslant n p /(n-2)$, alors pour tout $\alpha \in\{1, \ldots, n-1\}, \nabla \tilde{u}_{\mathrm{a}}$ appartient à $L^{k(s)}\left(\mathbb{R}_{+}^{n} \cap B(0, r), \mathbb{R}^{m n}\right)$ avec $k(s)=2 s /(s+2-p)$. (On pose $n p /(n-2)=+\infty$ si $n=2) .\left(\nabla \tilde{u}_{\alpha}\right.$ désigne le gradient de la dérivée partielle $\tilde{u}_{\mathrm{o}}$ ).

Proposition 3.4: Si une solution $u$ de (3.l) appartient $\dot{a} W^{i, s}\left(V, \mathbb{R}^{m}\right)$, avec $p \leqslant 2$ et $p \leqslant s \leqslant n p /(n-2)$, alors $u$ appartient à $W^{2, k(s)}\left(V, \mathbb{R}^{m}\right)$, avec $k(s)=2 s /(s+2-p)$. De plus, il existe une constante $C$ indépendante de $k(s)$ telle que l'on ait:

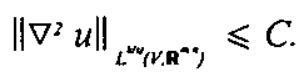

Remarqe: Pour tout $s$ vérifiant $p \leqslant s \leqslant n p /(n-2)$, on a $p \leqslant k(s) \leqslant n p /(n-2+p)$.

Commentaires sur la preuve de la proposition 3.3 .

L'estimation des dérivées partielles secondes $\tilde{u}_{\mathrm{ap}}$ avec $(\alpha, \beta) \neq(n, n)$ s'obtient à l'aide de la méthode des translations de Nirenberg. Le détail des calculs liés à cette méthode étant donné dans [10], dans le but d'alléger la rédaction, on procèdera ici de façon formelle en remplaçant les quotients diffèrentiels $\Delta_{-h}^{r}$ et $\Delta_{h}^{r}$ par les dérivées partielles $-\frac{\partial}{\partial y_{\gamma}}$ et $\frac{\partial}{\partial y_{\gamma}}$, avec $\Delta_{h}^{r} v(y)=$ $\left[v\left(y+h e_{\gamma}\right)-v(y)\right] h^{-1}$, où $h \in \mathbb{R}^{*}$ et $e_{\gamma}$ est un vecteur de la base canonique de $\mathbb{R}^{\mathrm{n}}$. La preuve de la proposition 3.3 se décompose en quatre étapes.

- Dans la première étape, on substitue $-\frac{\partial}{\partial y_{y}}\left(\frac{\partial}{\partial y_{\gamma}} \tilde{u}(y) \xi(y)\right)$ à $\tilde{\Phi}$ dans $(3.3)$, 
$0 \leqslant \gamma \leqslant n-1$, et on développe le calcul de façon à isoler le terme $A_{i}^{\mathrm{a} \beta}\left(y . T^{\delta}(y) \tilde{u}_{\delta}(y)\right) T_{\xi}^{\mathrm{b}} \tilde{u}_{\delta \gamma} T_{\mathrm{a}}^{\mathrm{b}} \tilde{u}_{b \gamma}^{i} \xi$. ( $\xi$ est une fonction à support dans $\mathbb{R}_{+}^{\mathrm{n}} \cap B(0, R)$ ).

- Dans la deuxième étape, par une minoration de convexité et par des majorations découlant de l'inégalité de Hölder et des différentes estimations de $\left(T_{a}^{\mathrm{b}}\right)$, on obtient:

$\int \mathbb{R}_{+}^{n}\left|T_{\mathrm{a}}^{\mathrm{b}} \tilde{u}_{\delta}\right|^{\mathrm{p}-2 \xi \xi(1-2 / k)}\left|T_{\mathrm{a}}^{\mathrm{b}} \tilde{u}_{6} \xi^{1 / k \mathrm{k}}\right|^{2} d y \leqslant C_{2}\left\|T_{\mathrm{a}}^{\delta} \tilde{u}_{\delta} \xi^{1 / k}\right\|_{\mathrm{p}}+C_{3}$, ou $C_{2}$ et $C_{3}$ ne dépendent pas de $k=k(s)$.

- Dans la troisième étape, avec l'inégalité de Hölder d'exposant négatif, on démontre que $\tilde{u}_{\alpha \beta} \in \mathrm{L}^{k(s)}\left(\mathbb{R}_{+}^{\mathrm{n}} \cap B(0, r), \mathbb{R}^{\mathrm{m}}\right)$ pour tout $(\alpha, \beta) \neq(n, n)$. On établira de plus une estimation de $\left\|\hat{u}_{\alpha \beta}\right\|_{L^{4+1}}$ que nous utiliserons dans la preuve de la proposition 3.4 .

Remarque: Le fait de remplacer les quotients différentiels par les dérivées partielles correspondantes simplifie les calculs, notamment, l'expression

$$
\int_{0}^{1} A_{i}^{\mathrm{a}, \beta}\left(y,(1-t) T^{\mathrm{s}}(y) \tilde{u}_{\delta}(y)+t T^{\mathrm{s}}\left(y+h e_{\gamma}\right) \tilde{u}_{\delta}\left(y+h e_{\gamma}\right)\right) d t .
$$

où $e_{\mathrm{r}}$ est un vecteur unitaire de direction parallèle à la frontière de $\mathbb{R}_{+}^{\mathrm{n}}$, est remplacée par:

$$
A_{i j}^{\mathrm{a} \beta}\left(y, T^{\mathrm{B}}(y) \tilde{u}_{\delta}(v)\right) \text {. }
$$

De façon à montrer que cette simplification n'a rien d'abusif, nous détaillerons à la quatrième étape la majoration la plus délicate à traiter dans la méthode des quotients différentiels.

Preuve de la Proposition 3.3.

lère étape: On remplace $\tilde{\Phi}$ dans (3.3) par $-\frac{\partial}{\partial y_{\gamma}}\left(\frac{\partial}{\partial y_{\gamma}}-\tilde{u} \xi\right)$ où $\xi$ est de classe $C^{2}$ et vérifie supp $\xi \subset B(0, R)$. Dans la preuve de la proposition $3.3, \gamma$ appartient à $\{1, \ldots, n-1\}$ et i] n'y a pas de sommation sur cet indice. On a:

$$
\begin{gathered}
\tilde{\Phi}_{b}=-\frac{\partial}{\partial \bar{y}_{\gamma}}\left(\tilde{u}_{5 \gamma} \xi\right)-\frac{\partial}{\partial y_{\gamma}}\left(\tilde{u}_{\gamma} \xi_{b}\right) \text { et } \\
\frac{\partial}{\partial y_{\gamma}}\left(\tilde{u}_{\delta \gamma} T^{\delta} \xi\right)=\frac{\partial}{\partial y_{\gamma}}\left(\tilde{u}_{\delta \gamma} \xi\right) T^{\delta}+\tilde{u}_{5 \gamma} \frac{\partial}{\partial y_{\gamma}}\left(T^{5}\right) \xi,
\end{gathered}
$$


soit encore:

$$
\begin{gathered}
T_{a}^{\mathrm{s}} \dot{\Phi}_{\delta}=-\frac{\partial}{\partial y_{\gamma}}\left(\tilde{u}_{\delta \gamma} T_{\mathrm{a}}^{\mathrm{b}} \xi\right)+\dot{u}_{\delta y} \xi T_{a y}^{\mathrm{b}}, \quad \text { avec } \\
T_{a \gamma \gamma}^{\mathrm{s}}=\frac{\partial}{\partial \bar{y}_{\gamma}} T_{\alpha}^{\delta}(y) .
\end{gathered}
$$

On pose:

$A_{,}()=.A_{i}^{a}\left(y, T^{\mathrm{b}}(y) \vec{u}_{\mathrm{a}}(y)\right)$, (on utilisera plus loin le même type de notation pour $A_{i \gamma}^{\mathrm{a}}$ et $\left.A_{i j}^{\mathrm{a}}\right)$,

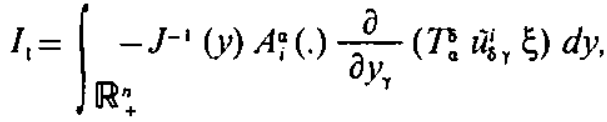

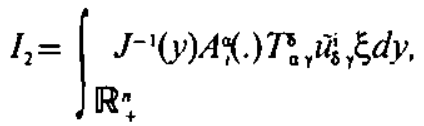

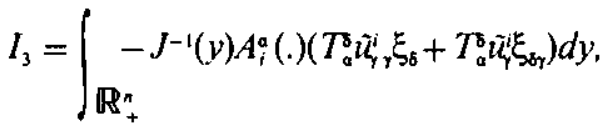

$$
\begin{aligned}
& I_{4}=\int_{\mathbb{R}_{+}^{n}}-\tilde{f}^{i}(y, \hat{u})\left(\hat{u}_{\gamma}^{i} \xi+\hat{u}_{\gamma}^{i} \xi_{\gamma}\right) d y,
\end{aligned}
$$

Le remplacement de $\tilde{\Phi}$ dans (3.3) par $-\frac{\partial}{\partial y_{\gamma}}\left(\tilde{u}_{\gamma} \xi\right)$ donne donc:

$$
I_{1}+I_{2}+I_{3}=I_{4}
$$

Par intégration par parties par rapport à $y_{\gamma}$ dans $I_{1}$, on obtient:

$$
\begin{aligned}
& I_{1}=I_{5}+I_{6}+I_{1}+I_{3}, \quad \text { avec }
\end{aligned}
$$

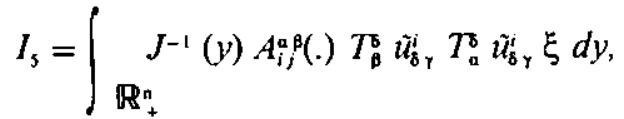

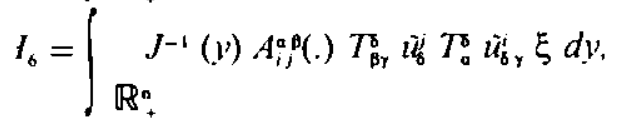

$$
\begin{aligned}
& I_{7}=\int J_{\mathbb{R}_{+}^{\mathrm{n}}}^{J^{-1}}(y) A_{i \gamma}^{\mathrm{a}}(.) T_{\alpha}^{\mathrm{b}} \tilde{u}_{\delta_{Y}}^{i} \xi d y, \\
& I_{\mathrm{s}}=\iint_{\mathbb{R}_{+}^{\mathrm{a}}} J_{r}^{-1}(y) A_{i}^{\mathrm{a}}(.) T_{\mathrm{a}}^{\mathrm{s}} \tilde{u}_{\mathrm{b}}^{\mathrm{i}} \xi d y
\end{aligned}
$$


On a donc:

$$
\left|I_{5}\right| \leqslant\left|I_{2}\right|+\left|I_{3}\right|+\left|I_{4}\right|+\left|I_{6}\right|+\left|I_{7}\right|+\left|I_{8}\right|
$$

2 ème étape. On choisit $\xi$ vérifiant:

$$
\begin{gathered}
\xi(y)=1 \text { si } y \in B(0, r), \\
0 \leqslant \xi(y) \leqslant 1 \text { si } y \in B(0, R) \backslash B(0, r), \\
\xi(y)=0 \text { si } y \in B(0, R), \\
\forall y \in B(0, R), \mid \nabla \xi(y) \xi(y)^{-|A(s)| \leqslant b_{6},}
\end{gathered}
$$

où $b_{6}$ est une constante positive indépendante de $k(s)$. Un tel choix de fonction $\xi$ est toujours possible (cf. [10]), il suffit de poser $\xi(v)=\eta(|y|)$ avec $\eta(\rho)$ $=(\mathrm{R}-\rho)^{k^{*}}$ où $k^{\prime}=k(s) /(k(s)-1)$, dans un voisinage à gauche de $R$.

On pose dans la suite $k=k(s)$.

Avec l'jnégalité de Hölder, on obtient les majorations suivantes:

$$
\left|I_{2}\right| \leqslant\|A,(.)\|_{p}\left\|J^{-1} T_{a}^{5} \tilde{u}_{b \gamma} \xi\right\|_{p}\left\|T_{a r}^{5}\left(T_{a}^{\delta}\right)^{-1}\right\|_{\infty} \text { où }\left(T_{a \gamma}^{\delta}\right)\left(T_{a}^{8}\right)^{-1}
$$

est un produit matriciel, $\left(T_{\alpha}^{5}\right)^{-1}$ désignant l'inverse de la matrice ( $7_{\mathrm{a}}^{\mathrm{b}}$ ).

Compte tenu de (3.4), (3.5) et (3.9), on a:

$$
\left|I_{3}\right| \leqslant\|A,(.)\|_{p},\left\{\left\|J^{-1} T^{Y} \tilde{u}_{n} \xi^{3 / k}\right\|_{p} b_{l}^{-1} b_{2} b_{b}+\left\|T_{\mathbf{a}}^{\mathbf{b}}\right\|_{\infty}\|\nabla \tilde{u}\|_{\mathrm{p}}\left\|\xi_{\delta_{\mathrm{r}}}\right\|_{\infty}\right\} .
$$

Compte tenu de (3.4) et (3.7), on a:

$$
\left|I_{4}\right| \leqslant\|\tilde{f}\|_{p},\left\{\left\|J^{-1} T^{r} \tilde{u}_{\gamma} \xi\right\|_{p} b_{1}^{-} b_{4}^{-1}+\left\|\tilde{u}_{\gamma}\right\|_{p},\|\nabla \xi\|_{\infty}\right\}
$$

On aussi:

$$
\begin{aligned}
& \left|I_{1}\right| \leqslant\left\|A_{, \gamma}^{a}(.)\right\|_{p^{\prime}}\left\|J^{-1} 7^{\delta} \dot{u}_{\delta}, \xi\right\|_{p}, \\
& \left|I_{s}\right| \leqslant\left\|A_{,}^{a}(.)\right\|_{D^{\prime}}\left\|J^{-1} T^{\delta} \dot{u}_{\delta} \xi\right\|_{p} .
\end{aligned}
$$

Compte-tenu de l'hypothèse (2.6), on a:

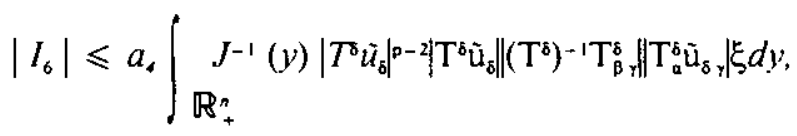

et avec l'hypothèse (2.7), on obtient: 


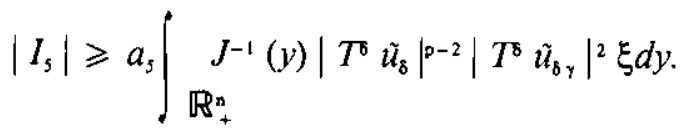

De (3.8), (3.10) et (3.11), par des majorations classiques, on obtient:

$$
I_{9}=\int \underset{\mathbb{R}_{+}^{\mathrm{n}}}{J^{-1}}\left|T^{\delta} \tilde{\mathrm{u}}_{8}\right|^{\mathrm{p}-2}\left|\mathrm{~T}^{\delta} \tilde{\mathrm{u}}_{\delta \gamma}\right|^{2} \xi d y \leqslant\left|I_{2}\right|+\left|I_{3}\right|+\left|I_{4}\right|+\left|I_{\gamma}\right|+\left|I_{8}\right|+\left|I_{10}\right|
$$

avec

$$
I_{10}=c_{1} \int_{\mathbb{R}_{+}^{n}} J^{-1}(y)\left|T^{\mathrm{\delta}} u_{\delta}\right|^{p}\left|\left(T^{5}\right)^{-1} T_{\beta}^{\mathrm{s}}\right|^{2} \xi d y \text {. }
$$

Compte tenu des estimations de $\left|I_{2}\right|,\left|I_{3}\right|,\left|I_{4}\right|,\left|I_{7}\right|,\left|I_{3}\right|$, de l'inégalité $|\xi| \leqslant$ $\left|\xi^{1 / k}\right|$, des hypothèses (2.4), (2.2), (2.5), de (3.8) et (3.7), on déduit:

$$
\int T_{\mathbb{R}_{+}^{\mathrm{n}}}\left|T^{\delta} \tilde{u}_{\delta}\right|^{p-2}\left(\xi^{1-2 / k}\right)\left|T^{\delta} \tilde{u}_{\delta \gamma} \xi^{1 / k}\right|^{2} d y \leqslant c_{2}\left\|T^{\delta} \tilde{u}_{\delta \gamma} \xi^{1 / k}\right\|_{p}+c_{3}
$$

où $c_{2}$ et $c_{3}$ dépendent de $\|\nabla u\|_{\mathrm{p}}$ et $\|u\|_{p^{2}}$ mais ne dépendent pas de $k$.

3ème étape: Avec l'inégalité đe Hölder d'exposant négatif (lemme 2.1), on obtient:

$$
\left\{\int_{\mathbb{R}_{+}^{\mathrm{n}}}\left|T^{\mathrm{s}} \hat{u}_{\delta \gamma} \xi^{1 / k}\right|^{k} d y\right\}^{2 / k} \leqslant c_{4}\left\|T^{\mathrm{\delta}} \dot{u}_{5} \xi\right\|_{s}^{2-\mathrm{p}}\left(\left\|T^{\mathrm{\delta}} \tilde{u}_{5 \gamma} \xi^{1 / k}\right\|_{\mathrm{p}}+1\right),
$$

soit encore:

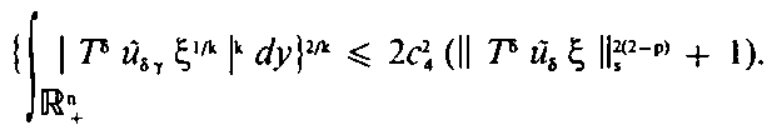

Compte tenu de (3.4) et (3.5), on obtient:

$$
\tilde{u}_{8 \gamma} \in \mathrm{L}^{\mathrm{k}}\left(\mathbb{R}_{+}^{\mathrm{n}} \cap B(0, r), \mathbb{R}^{\mathrm{m}}\right) .
$$

La proposition 3.3 est donc démontrée. De plus, de (3.12), on déduit:

$$
\left\{\int_{\mathbb{R}_{+}^{\mathrm{n}}}\left|\tilde{u}_{6 r} \xi^{1 / k}\right|^{k} d y\right\}^{2 / k} \leqslant c_{3}\left(\|\nabla \tilde{u} \xi\|_{s}^{2(2-p)}+1\right),
$$

pour tout $(\delta, \gamma) \neq(n, n)$ et avec $c_{s}$ indépendante de $k$.

4ème étape: Dans la méthode de Nirenberg, l'opérateur de dérivation partielle $\frac{\partial}{\partial y_{\gamma}}$ est remplacé par l'opérateur $\Delta_{h}^{\gamma}$ défini par: 
$\Delta_{\mathrm{h}}^{\gamma} v(y)=\left[v\left(y+h e_{\gamma}\right)-v(y)\right] h^{-1}$, où $h \in \mathbb{R}^{*}$ et $e_{\gamma}$ est un vecteur unitaire. $\left(\left(e_{y}\right)_{1<r<n}\right.$ est la base canonique de $\left.\mathbb{R}^{n}\right)$. La méthode consiste à remplacer $\tilde{\Phi}$ dans (3.3) par $\Delta_{-\mathrm{h}}^{\gamma}\left(\Delta_{\mathrm{h}}^{\gamma} \tilde{u} \xi\right)$ et à transposer $\Delta_{-\mathrm{h}}^{\gamma}$. On obtient alors (cf. [10]):

$$
\iint_{\mathbb{R}_{+}^{\mathrm{n}}} \Delta_{\mathrm{h}}\left(J^{-1}(y) A_{\mathrm{j}}^{\mathrm{a}}\left(\mathrm{y}, \mathrm{T}^{\mathrm{\delta}}(\mathrm{y}) \tilde{\mathrm{u}}_{5}(\mathrm{y})\right) \mathrm{T}_{\mathrm{a}}^{\mathrm{s}}(\mathrm{y})\right)\left(\Delta_{\mathrm{h}} \tilde{\mathrm{u}}_{8}(\mathrm{y}) \xi(\mathrm{y})+\Delta_{\mathrm{h}} \tilde{\mathrm{u}}(\mathrm{y}) \xi_{\delta}(\mathrm{y})\right) d \mathrm{y}
$$

dans le premier membre de l'équation (3.3). ( On a posé $\Delta_{h}^{r}=\Delta_{h}$ ). Le développement des calculs conduit à différents termes, examinions uniquement le plus délicat à traiter:

$$
\begin{aligned}
& J_{1}=\int_{\mathbb{R}^{\mathrm{n}}{ }_{+}} J^{-1}(y)\left[A_{\mathrm{i}}^{\mathrm{q}}\left(y, T^{\mathrm{p}}\left(y+h e_{\gamma}\right) \check{u}_{\delta}\left(y+h e_{\gamma}\right)\right)\right. \\
& \left.-A_{\mathrm{i}}^{\mathrm{a}}\left(y, T^{\mathrm{b}}(y) \tilde{u}_{\delta}(y)\right)\right] T_{\mathrm{a}}^{\mathrm{b}}(y) \Delta_{\mathrm{a}} \tilde{\mathrm{u}}_{\mathrm{b}}^{\mathrm{j}}(y) \xi(y) d y .
\end{aligned}
$$

Remarquons de plus que l'intégrande de $J_{1}$ est nulle si $\tilde{u}_{\delta}\left(y+h e_{\gamma}\right)=\tilde{u}_{\delta}(y)=$ $0_{\mathrm{mn}}$. On pose: $S=\left\{y \mid \tilde{u}_{\bar{\delta}}\left(y+h e_{\uparrow}\right)=\tilde{u}_{\delta}(y)=0_{\mathrm{mn}}\right\}$. Par un calcul classique, on obtient:

$$
\begin{aligned}
& \mathrm{J}_{\mathrm{L}}=\int_{\mathbb{R}_{+}^{\mathrm{a}} \backslash \mathrm{S}}^{J^{-1}(y)} \int_{0}^{1} A_{\mathrm{i}, \mathrm{\alpha}}^{\mathrm{a} \beta}\left(y,(1-t) T^{\mathrm{b}}(y) \tilde{u}_{\delta}(y)+t T^{\mathrm{b}}\left(y+h e_{\gamma}\right) \tilde{u}_{8}\left(y+h e_{\gamma}\right)\right) d t \\
& \times \Delta_{\mathrm{h}}\left(T_{\beta}^{\mathrm{s}}(y) \tilde{u_{\mathrm{b}}^{i}}(y)\right) T_{\beta}^{\mathrm{s}}(y) \Delta_{\mathrm{h}} \tilde{u}_{\mathrm{b}}^{i}(y) \xi(y) d y .
\end{aligned}
$$

On pose:

$$
\tilde{A}_{\mathrm{ij}}^{\mathrm{\alpha} \beta}(t)=A_{i j}^{\mathrm{\alpha} \beta}\left(y,(1-t) T^{\delta}(y) \tilde{u}_{\delta}(y)+t T^{\delta}\left(y+h e_{\gamma}\right) \tilde{u}_{\delta}\left(y+h e_{\gamma}\right)\right) .
$$

On a:

$$
J_{1}=J_{2}+J_{3}
$$

avec

$$
\begin{aligned}
& J_{2}=\int_{\mathbb{R}_{+}^{\mathrm{a}} \backslash \mathrm{S}}^{J^{-1}(y)} \int_{0}^{1} \tilde{A}_{\mathrm{ij}}^{\mathrm{a}}(t) d t \Delta_{\mathrm{h}} T_{\beta}^{\mathrm{b}}(y) \tilde{w}_{\mathrm{b}}^{\mathrm{j}}\left(y+h e_{\gamma}\right) T_{\mathrm{a}}^{\mathrm{b}}(y) \Delta_{\mathrm{h}} \tilde{u}_{\mathrm{z}}(y) \xi(y) d y,
\end{aligned}
$$

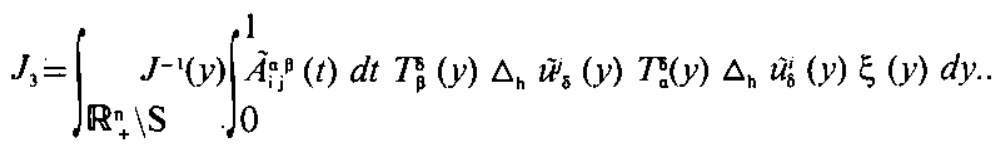

De l'hypothèse (2.7) et du lemme 2.2 , on déduit l'existence d'une constante positive $c_{6}$ telle que: 


$$
J_{3} \geqslant c_{6}\left\{\begin{array}{c}
J_{\mathbb{R}_{+}^{\mathrm{n}} \backslash S} J^{-1}(y)\left(\left|T^{\mathrm{\delta}}(y) \tilde{u}_{\delta}(y)\right|+\left|T^{\mathrm{\delta}}\left(y+h e_{\gamma}\right) \tilde{u}_{\delta}\left(y+h e_{\gamma}\right)\right|\right)^{p-2} \\
\times\left|T^{\mathrm{D}}(y) \Delta_{\mathrm{h}} \tilde{u}_{\delta}(y)\right|^{2} \xi(y) d y .
\end{array}\right.
$$

De l'hypothèse (2.6) et du lemme 2.2 , on déduit l'existence d'une constante $c_{7}>0$ telles que:

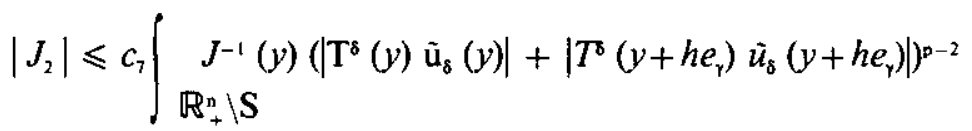

$$
\begin{aligned}
& \left|T^{\mathrm{v}}(y) \Delta_{\mathrm{h}} \tilde{u}_{\mathrm{s}}(y)\right|\left|\Delta_{\mathrm{h}} T^{\mathrm{\nabla}}(y) \tilde{\mathrm{u}}_{\mathrm{s}}\left(y+h e_{\mathrm{\gamma}}\right)\right| \xi(y) d y .
\end{aligned}
$$

Avec une inégalité du type:

$$
g^{2}\left(\dot{y)}-g(y) h(y) \geqslant \frac{1}{2} g^{2}(y)-\frac{1}{2} h^{2}(y)\right.
$$

et par des majorations classiques, on obtiendra les expressions suivantes:

$$
\begin{gathered}
J_{4}=\int \underset{\mathbb{R}_{+}^{\mathrm{n}} \backslash \mathrm{S}}{J^{-1}(y)\left(\left|T^{5}(y) \tilde{u}_{\delta}(y)\right|+\left|T^{5}\left(y+h e_{\gamma}\right) \tilde{u}_{\delta}\left(y+h e_{\gamma}\right)\right|\right)^{\mathrm{p}-2}} \\
\times\left|T^{\mathrm{D}}(y) \Delta_{\mathrm{h}} \tilde{u}_{5}(y)\right|^{2} \xi(y) d y
\end{gathered}
$$

et

$$
\begin{aligned}
J_{s}= & \int \begin{array}{c}
J^{-1}(y)\left(\gamma^{\delta}(y) u_{\delta}(y)|+| T^{\delta}\left(y+h e_{\gamma}\right) u_{\delta}\left(y+h e_{\gamma}\right) \mid\right)^{p-2} \\
\mathbb{R}^{n} \mathrm{~S}
\end{array} \\
& \times\left|T^{\mathrm{s}}\left(y+h e_{\gamma}\right) \tilde{u}_{\delta}\left(y+h e_{\gamma}\right)\right|^{2}\left|\left(T^{8}\left(y+h e_{\gamma}\right)\right)^{-1}\right|^{2}\left|\Delta_{\mathrm{h}} T^{8}(y)\right|^{2} \xi(y) d y .
\end{aligned}
$$

Les intégrales $J_{4}$ et $J_{5}$ remplacent respectivement (à des constantes multiplicatives près) les expressions $I_{9}$ et $I_{10}$ des calculs précédents. La fin est alors classique et les autres difficultés liées à l'application de la méthode de Nirenberg sont détaillées dans [10].

Preuve de la Proposition 3.4. Soit $u$ une solution de (3.1) et $\tilde{u}$ la solution de (3.3) définie en (3.2). Si $u$ appartient à $\mathrm{W}^{1, s}\left(V, \mathbb{R}^{\mathrm{m}}\right)$, $\tilde{u}$ appartient â $\mathrm{W}^{\mathrm{l}, \mathrm{s}}\left(\mathbb{R}_{+}^{\mathrm{n}} \cap B(0, r), \mathbb{R}^{\mathrm{m}}\right)$ et de la proposition 3.3 , on déduit que pour tout $\alpha$ de $\{1, \ldots, n-1\}, \nabla \tilde{u}_{\mathrm{a}} \in \mathrm{L}^{\mathrm{k}(s)}\left(\mathbb{R}_{+}^{\mathrm{n}} \cap B(0, r), \mathbb{R}^{\mathrm{m} n}\right)$.

Nous allons montrer que $\nabla^{2} \mathfrak{u}$ appartient à $L^{k(s)}$ et obtenir une estimation de

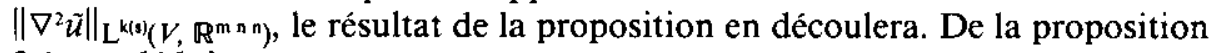
3.1 , on déduit que

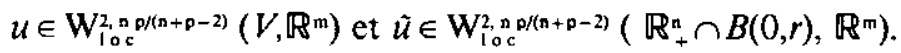


Les fonctions $u$ et $\tilde{u}$ admettent donc des dérivées partielles premières et secondes au sens classique presque partout (cf. [2] theorème 3.1). On note $\hat{B}=\left\{y \in \mathbb{R}^{n}{ }_{+} \cap B(0, r) \mid \tilde{u}\right.$ admet des dérivées partielles premières et secondes en $y\}, N=|y \in \hat{B}| \nabla \tilde{u}(y)=0_{\mathrm{m}} \mid$. On va estimer séparément $\nabla^{2} \tilde{u}$ sur $\bar{N}$ et sur $\left(\mathbb{R}^{\mathrm{n}}{ }_{+} \cap B(0, r)\right) \backslash \bar{N}(\bar{N}$ désignant l'adhérence de $N)$.

lère étape: Pour estimer $\nabla^{2} \tilde{u}$ sur $\bar{N}$, on considère l'en'semble suivant: $\hat{N}=\hat{N} \cap \hat{B}$. On a Mes $\hat{N}=$ Mes $\bar{N}$. De plus, $\nabla \tilde{u}$ est dérivable et donc aussi continu sur $\hat{N}$. On en déduit que:

$$
\nabla \tilde{u}(y)=0_{m n} \text { si } y \in \hat{N} .
$$

On a donc: $\nabla^{2} \tilde{u}(y)=0_{\mathrm{m} n \mathrm{n}}$ pour presque tout $y \in \bar{N}$ (cf, lemme 7.7 [20]).

2ème étape. Estimation de $\nabla^{2} u$ ù sur $B^{+} \backslash \bar{N}$ (avec $B^{+}=\mathbb{R}_{+}^{n} \cap B(0, r)$ ). De l'équation (3.3), on déduit que, pour presque tout y de $B^{+} \backslash \hat{N}$, on a:

$$
-\frac{\partial}{\partial y_{\delta}}\left[J^{-1}(y) \text { A }\left(y, T^{5}(y) \tilde{u}_{\delta}(y)\right) T_{a}^{8}(y)\right]=J^{-1}(y) \tilde{f}^{\prime}(y, \tilde{u}(y))
$$

au sens des distributions.

Soit y un point de $\left(B^{+} \backslash \bar{N}\right) \cap \hat{B}, \nabla \tilde{u}$ étant continue en y, il existe un voisinage $\theta$ de y sur lequel $\nabla \dot{u}$ est bornée. On peut donc montrer par des arguments classiques que sur $\theta$, on a:

$$
\begin{aligned}
& \frac{\partial}{\partial y_{\delta}}\left[J^{-1}(y) \operatorname{A}_{j}\left(y, T^{\delta}(y) \tilde{u}_{\delta}(y)\right) T_{a}^{\sigma}(y)\right] \\
& =J_{\tilde{b}}^{-1}(y) A_{i}^{\alpha}(.) T_{a}^{b}(y)+J^{-1}(y) A_{1 b}^{\delta}(.) T_{a}^{b}(y)+
\end{aligned}
$$

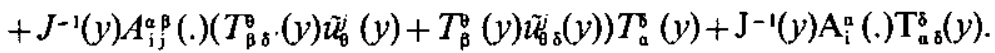

De (3.14) et (3.15), on déduit:

$$
\begin{aligned}
& J^{-1}(y) A_{\mathrm{ij}}^{\mathrm{\alpha}}(.) T_{\beta}^{n}(y) \tilde{\mathrm{u}}_{\mathrm{in}}^{j}(y) T_{\mathrm{a}}^{n}(y)= \\
& =-J_{\mathrm{\delta}}^{-1} A_{\mathrm{i}}^{\mathrm{q}}(.) T_{\mathrm{a}}^{\mathrm{s}}-J^{-1} A_{\mathrm{i} \delta}^{\mathrm{q}}(.) T_{\mathrm{a}}^{\mathrm{s}}-J^{-1} A_{\mathrm{i} j}^{\mathrm{\alpha} \beta} T_{\mathrm{\beta} \delta}^{\mathrm{q}} \tilde{\mathrm{u}}_{\mathrm{b}}^{j} T_{\mathrm{a}}^{\mathrm{g}}
\end{aligned}
$$

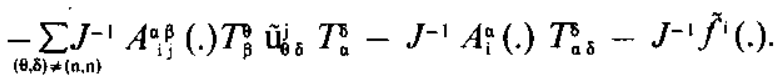

En multipliant les deux membres de (3.16) par $\tilde{u}_{n}^{i}{ }_{n}^{i}$ et en sommant sur $i$, avec les hypothèses (2.4) à (2.7), on obtient:

$$
\left|T^{n} \tilde{u}_{n n}\right|^{2} \leqslant c_{g}\left|\tilde{u}_{n n}\right|\left(|\nabla \tilde{u}|+\sum_{(\theta, \delta) *(\mathrm{a}, \mathrm{n})}\left|\tilde{u}_{\theta \delta}\right|+|\nabla \tilde{u}|^{2-\phi|\tilde{f}(.)|) .}\right.
$$


Compte tenu de (3.4), par des majorations classiques, on a:

(3.17) $\left|\tilde{\mathrm{u}}_{\mathrm{n} n}\right| \leqslant \mathrm{c}_{10}\left[|\nabla \tilde{\mathrm{u}}|+\sum_{\gamma=1}^{a-1}\left|\nabla \tilde{\mathrm{u}}_{\gamma}\right|+\left.|\tilde{f}().| \nabla \tilde{\mathrm{u}}\right|^{2-\mathrm{p}}\right]$.

Avec l'hypothese (2.2) et (3.13), on montre que $\tilde{u}_{n} \in \mathrm{L}^{\mathrm{k}(\mathrm{s})}$ et qu'il existe une constante $c_{11}$, indépendante $k$, pour laquelle on a:

$$
\iint_{\Omega}\left|\nabla^{2} \tilde{u}(y) \xi(y)^{1 / \alpha \mid k} \mathrm{dy}\right|^{2 / k} \leqslant c_{11}\left(\|\nabla \tilde{u} \xi\|_{s}^{2(2-p)}+1\right) .
$$

De plus, avec l'inégalité de Sobolev, on a:

$\|\nabla \tilde{u} \xi\|_{s} \leqslant c(s)\left(\left\|\nabla^{2} \tilde{u} \xi\right\|_{s .}+\|\nabla \tilde{u} \nabla \xi\|_{w}\right.$, dés que $s \leqslant \frac{n}{n-1}$ et avec $s_{*}=\frac{n s}{n+s}$,

$c(s)$ étant la constante de l'inégalité de Sobolev pour les espaces considérés.

De $s \leqslant \frac{n p}{n-2}$, on déduit $s * \leqslant k(s) \leqslant 2$. Il est donc possible de majorer $c(s)$ et $\|\nabla \tilde{u} \nabla \xi\|_{S_{s}}$, indépendamment de $s$, finallement, avec (3.18), on obtient: $\iint_{\Omega}\left|\nabla^{2} \tilde{u}(y) \xi(y)^{1 / k \mid k} d y\right|^{2 / \pi} \leqslant c_{12}\left(\left\|\nabla^{2} \tilde{u} \xi^{1 / k}\right\|_{L^{2(2-p)}}+1\right)$.

Comme on a $s_{*} \leqslant k$ et $2(2-p)<2$, on obtient:

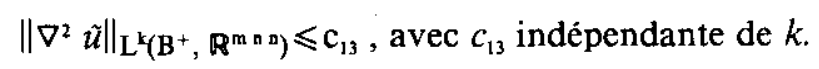

Preuve du théorème 3.2: On reprend ici la preuve du théorème 5.1 de [10].

lère étape. De la proposition 3.4 et du théorème d'injection de Sobolev, on déduit que si une solution $u$ de (3.1) appartient à $W^{1.0}\left(V, \mathbb{R}^{m}\right)$, alors $u$ appartient

à $W^{1, k s}\left(V, \mathbb{R}^{\mathrm{m}}\right)$ avec $l(s)=\frac{2 n s}{n(s-p+2)-2 s}$.

2ème étape. On vérifie facilement que la suite définie par $s_{0}=p$ et $s_{\mathrm{j}+1}=$ $\frac{2 s_{j} n}{n\left(s_{j}-p+2\right)-2 s_{j}}$ converge vers $\frac{n p}{n-2}$ si $n>2$ et vers $+\infty$ si $n=2$.

3 ème étape. On démontre, par récurrence sur $j$, que l'on a: 


$$
\forall j \in \mathbb{N}, \mathrm{u} \in \mathbb{W}^{1, s_{\mathrm{j}}}\left(V, \mathbb{R}^{m}\right) \text { et } u \in \mathbb{W}^{\left.2, \mathrm{k}_{\mathrm{j}}\right)}\left(V, \mathbb{R}^{\mathrm{n}}\right) .
$$

4ème étape. Le théorème découle maintenant d'un argument de recouvrement, de ce que $\lim _{j \rightarrow+\infty} k\left(s_{j}\right)=\frac{n p}{n+p-2}$ et de ce que:

$$
\forall j \in \mathbb{N},\left\|\nabla^{2} \tilde{\mathbf{u}}\right\|_{L^{k\left(s_{j}\right)}} \leqslant c_{13} .
$$

\section{LEMMES TECHNIQUES}

Dans [10], les résultats de régularité pour $p>2$ sont obtenus par dualité. Nous procéderons au paragraphe 5 de manière analogue. Nous introduisons pour celà quelques notions préliminaires et nous établissons des résultats techniques utiles dans la suite.

Etant donné que $p$ est supérieur à 2, nous pouvons supposer que les fonctions $a_{i}^{a}$ appartiennent à $C^{1}\left(\mathbb{R}^{n} \times \mathbb{R}^{m}\right.$ n) et que les estimations (2.4) à (2.7) sont vérifiées pour tout $(x, v)$ de $\mathbb{R}^{\mathrm{n}} \times \mathbb{R}^{\mathrm{m} n}$. Si l'on pose:

$$
a(x, v)=\int_{0}^{1}(1-t) a_{i j}^{\alpha}(x, t) v_{\alpha}^{\alpha} v_{\beta}^{j} d t,
$$

de la définition de $a_{i j}^{\alpha \beta}$ de la condition de symétrie $a_{i j}^{\alpha \beta}=a_{j ;}^{\beta, ~ e t ~ d e s ~ e s t i m a t i o n s ~}$ (2.4) à (2.7), on déduit:

$$
a \in C^{l}\left(\mathbb{R}^{n} \times \mathbb{R}^{m n}\right),
$$

$\frac{\partial}{\partial v_{\beta}^{j}} a(x, v)=a_{j}^{\mathrm{\beta}}(x, v)$ pour tout $j$, tout $\beta$, tout $x$, tout $y$,

et $a(x,$.$) est strictement convexe.$

Nous noterons $b(x,$.$) la polaire de a(x,$.$) au sens de la définition de [6]. Nous$ allons démontrer les lemmes suivants.

Lemme 4.1: La fonction b vérifie les résultats de régularité suivants:

$$
\begin{gathered}
b \in C^{\prime}\left(\mathbb{R}^{\mathrm{n}} \times\left(\mathbb{R}^{\mathrm{m} n} \backslash\left\{0_{\mathrm{m} \text { 。 }}\right\}\right)\right), \\
b_{\mathrm{i}}^{\mathrm{a}} \in C^{1}\left(\mathbb{R}^{\mathrm{n}} \times\left(\mathbb{R}^{\mathrm{m} \mathrm{n}} \backslash\left\{0_{\mathrm{m}}\right\}\right)\right) \text { où } b_{i}^{a}(x, q)=\frac{\partial}{\partial \ddot{q}_{i}^{a}} b(x, q) .
\end{gathered}
$$

Notations: On a posé $q=\left(q_{i}^{a}\right)_{\substack{1 \leq a \leq n \\ 1<i \leqslant m}}$. Dans la suite, on pose: 


$$
b_{i \gamma}^{\mathrm{a}}(x, q)=\frac{\partial}{\partial x_{\gamma}} b_{j}^{\mathrm{a}}(x, q) \text { et } b_{i j}^{\mathrm{a} \beta}(x, q)=\frac{\partial}{\partial q_{j}^{\beta}} b_{i}^{a}(x, q)
$$

Lemme 4.2: Il existe des constantes positives $b_{7, \ldots,}, b_{9}$ telles que, pour tout $x$ de $\Omega$, tout $q$ de $\mathbb{R}^{m n} \backslash\left\{0_{m \pi}\right\}$, tout $\chi$ de $\mathbb{R}^{m n}$, on ait:

$$
\begin{aligned}
& \left|b_{\mathrm{j}}^{a}(x, q)\right| \leqslant b_{7} \mid u^{\mathrm{p}^{\mathrm{p}^{\prime}-1}} \\
& \left|b_{i,}^{\kappa}(x, g)\right| \leqslant b_{7}|q|^{p^{2}-1} \\
& \left|b_{i j}^{\mathrm{a \beta}}(x, q)\right| \leqslant b_{8}|q|^{\mathrm{p}^{-2}} \\
& b_{i j}^{\alpha \beta}(x, q) \chi_{i}^{\alpha} \chi_{j}^{\beta} \geqslant\left. b_{9}\left|q^{p i-2}\right| \chi^{2}\right|^{2} .
\end{aligned}
$$

Preuve du lemme 4.1: Par définition de $b$, on a:

$$
b(x, q)=\operatorname{Sup}_{v \in \mathbb{R}^{\mathrm{m} \mathrm{n}}}(q v-a(x, v))
$$

La fonction $a\left(x_{\text {,. }}\right)$ est strictement convexe, de plus on a:

$$
a_{i}^{\alpha}(x, v)=\int_{0}^{1} a_{\mathrm{i} j}^{\alpha \beta}(x, t v) \nu_{\mathrm{j}}^{\mathrm{j}} d t
$$

De (4.7), on déduit que le supremum de $q v-a(x, v)$ est atteint au point $v$ vérifiant le système:

$$
a_{i}^{a}(x, v)=q_{i}^{a}
$$

On en déduit que si $q \neq 0_{m n}$, alors $v \neq 0_{m n}$. Des hypothèses $(2.3),(2.7)$ et du théorème d'inversion locale, on déduit que l'application de $\mathbb{R}^{\mathrm{n}} \times \mathbb{R}^{\mathrm{m} \mathrm{n}}$ qui à $(x, q)$ fait correspondre $v(x, q)$, où $v(x, q)$ est l'unique solution de (4.5), est de classe $C^{\prime}$ sur $\mathbb{R}^{n} \times\left(\mathbb{R}^{\mathrm{m} n} \backslash\left\{0_{\mathrm{m} \mathrm{n}}\right\}\right)$. De l'égalité: $b(x, q)=q v(x, q)-a(x, v(x, q))$, on déduit: $b \in C\left(\mathbb{R}^{n} \times\left(\mathbb{R}^{\mathrm{m} \square} \backslash\left\{0_{\mathrm{mn}}\right\}\right)\right)$. De plus, $b(x,$.$) étant la transformée$ de Legendre de $a(x$,$) , il est bien connu que l'on a \left(b_{\mathrm{i}}^{\mathrm{a}}(x, q)\right)_{\substack{1 \leq a \leq n \\ \operatorname{sic} \mathrm{m}}}=v(x, q)$. On a donc $b_{\mathrm{i}}^{\mathrm{a}} \in C^{\mathrm{y}}\left(\mathbb{R}^{\mathrm{n}} \times\left(\mathbb{R}^{\mathrm{m} n} \backslash\left\{0_{\mathrm{m} n}\right\}\right)\right)$.

Preuve du lemme 4.2. De l'égalité

$$
a_{\mathrm{i}}^{\mathrm{u}}(x, v)=\int_{0}^{1} a_{\mathrm{ij}}^{\alpha \beta}(x, t v) \nu_{\beta}^{j} d t
$$


et de (2.7), on déduit l'existence d'une constante positive $a_{8}$ telle que:

$$
\left|a_{\mathrm{i}}^{\mathrm{a}}(x, y)\right| \geqslant a_{s} \mid y^{\mathrm{p}-1} \text {. }
$$

On en déduit:

$$
|q|=\left|a_{\mathrm{i}}^{\alpha}\left(x,\left(b_{\mathrm{i}}^{\alpha}(x, q)\right)\right)\right| \geqslant a_{s}\left|b_{\mathrm{i}}^{\mathrm{a}}(x, q)\right|^{\mathrm{p}-1},
$$

soit encore:

$$
\left|b_{\mathrm{i}}^{\mathrm{a}}(x, q)\right| \leqslant a_{\mathrm{g}}{ }^{1 /(2)-p)}|q|^{\mathrm{p}^{\prime}-1} \text {. }
$$

Des propriétés de la transformée de Legendre, on déduit que si $v$ et $q$ satisfont (4.5), on a:

$$
\left(b_{i j}^{\alpha \beta}(x, q)\right)=\left(a_{i j}^{\alpha \beta}(x, v)\right)^{-1},
$$

où ( $\left.b_{i j}^{\alpha \beta}\right)$ et $\left(a_{i j}^{\alpha \beta}\right)$ sont considérées comme matrices de formes quadratiques sur $\mathbb{R}^{\mathrm{m}} \mathrm{n}$. Il existe une constante $c(n, m)$ ne dépendant que de $n$ et $m$ telle que: $\left|\mathrm{b}_{i j}^{\mathrm{a} \beta}(x, q)\right| \leqslant c(n, m) \Lambda\left(\mathrm{b}_{i j}^{\mathrm{a} \beta}(x, q)\right)$, oú $\Lambda\left(\mathrm{b}_{i j}^{\alpha \beta}(x, q)\right)$ est la plus grande valeur propre de $\left(b_{i j}^{\alpha \beta}(x, q)\right)$.

On a de plus: $\Lambda\left(b_{i j}^{\alpha \beta}(x, v)\right)=\left(\lambda\left(a_{i j}^{\alpha \beta}(x, v)\right)\right)^{-1} \leqslant a_{5}^{-1}|\psi|^{2-p}$, où $\lambda\left(a_{1 j}^{\mathrm{a \beta}}(x, v)\right)$ désigne la plus petite valeur propre de $a_{\mathrm{ij}}^{\mathrm{a}}(x, v)$. Finallement, on a:

$$
\left|b_{\mathrm{ij}}^{\mathrm{p}}(x, q)\right| \leqslant c(n, m) a_{3}^{-1}\left|b_{\mathrm{i}}^{\mathrm{a}}(x, q)\right|^{2-p} .
$$

Avec l'estimation précédente de $b_{\mathrm{j}}^{\mathrm{a}}$, on obtient:

$$
\left|b_{\mathrm{i} \mathrm{j}}^{\alpha \beta}(x, q)\right| \leqslant b_{\mathrm{g}}|q|^{(2-\mathrm{p})(\mathrm{p}-1)}=b_{\mathrm{z}}|q|^{\mathrm{p}^{\prime}-2},
$$

où $b_{8}$ dépend de $a_{5}, a_{8}$ et $c(n, m)$. En dérivant par rapport à $x_{r}$ l'identité:

$$
b_{\mathrm{i}}^{a}\left(x,\left(a_{\mathrm{i}}^{a}(x, v)\right)\right)=v,
$$

on obtient:

$$
b_{\mathrm{i} \gamma}^{\mathrm{a}}(x, q)+b_{\mathrm{i} j}^{a \beta}(x, q) a_{\mathrm{i \gamma}}^{\mathrm{a}}(x, v)=0 \text { avec } q_{\mathrm{i}}^{\mathrm{a}}=a_{\mathrm{i}}^{\mathrm{a}}(x, v) .
$$

On en déduit:

$$
\begin{gathered}
\left|b_{\mathrm{i \gamma}}^{\mathrm{a}}(x, q)\right| \leqslant b_{8}|q|^{p^{p}-2} a_{3}|v|^{p-1} \\
\leqslant b_{8^{\prime}} a_{3 \cdot} \cdot\left(b_{6}\right)^{p-1}|q|^{p^{\prime}-2}|q|^{\left(p^{p}-1\right)(p-1)}=b_{7}|q|^{p^{\prime}-1} \text {, }
\end{gathered}
$$


L'estimation (4.4) découle de (2.6) et de l'égalité:

$$
\lambda\left(b_{i j}^{\alpha \beta}(x, q)\right)=\left(\Lambda\left(a_{i j}^{a \beta}(x, v)\right)\right)^{-1},
$$

oú $\lambda\left(b_{i j}^{a f}\right)$ désigne la plus petite valeur propre de $\left(b_{i j}^{a p}\right)$ et $\Lambda\left(a_{i j}^{\alpha \mathbb{f}}\right)$ désigne la plus grande valeur propre de $\left(a_{\mathrm{i}}^{\mathrm{a}}\right)$.

\section{RESULTATS DE REGULARITE POUR $P>2$}

Comme dans [10], on pose la définition suivante.

Definition 5.1: On dit qu'une fonction $q$ de $L^{p^{\prime}}\left(\Omega, \mathbb{R}^{m n}\right)$ est une solution adjoin-

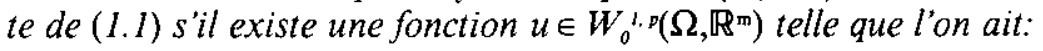

$$
\begin{aligned}
& q_{i}^{a}(x)=a_{i}^{a}(x, \nabla u(x)) \text { pour presque tout } x \text { de } \Omega, \\
& \frac{\partial}{\partial x_{\mathrm{a}}} q_{i}^{a}(x)=-f^{\prime}(x, u(x)) \text { dans }\left[D^{\prime}(V)\right]^{\mathrm{m}} .
\end{aligned}
$$

On dira alors que q est associée à $u$.

On a la

Proposition 5.2: Si une fonction $q$ de $L^{p^{\circ}}\left(\Omega, \mathbb{R}^{m}\right)$ est une solution adjointe de

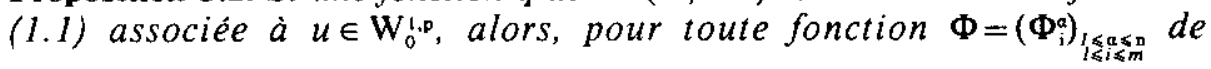
$L^{r}\left(\Omega, \mathbb{R}^{m n}\right)$ satisfaisant

$$
\frac{\partial}{\partial x_{\mathrm{a}}} \Phi_{\mathrm{i}}^{\mathrm{q}} \in \mathrm{L}^{\mathrm{p}}\left(\Omega, \mathbb{R}^{\mathrm{m}}\right)
$$

on $a$ :

$$
-\int_{\Omega} \frac{\partial}{\partial x_{\mathrm{a}}} \Phi_{i}^{q}(x) u^{u}(x) d x=\int_{\Omega} b_{i}^{\alpha}(x, q(x)) \Phi_{\mathrm{i}}^{\alpha}(x) d x .
$$

Le système (5.1.ii), (5.3) est appelé équation adjointe de (1.1).

Preuve: Par polarité, de (5.1.i), on déduit: $u_{\mathrm{a}}^{i}(x)=b_{i}^{a}(x, q(x))$, soit encore:

$$
\int_{\Omega} u_{\mathrm{i}}^{\mathrm{a}}(x) \boldsymbol{\Phi}_{\mathrm{i}}^{\alpha}(x) d x=\int_{\Omega} \mathrm{b}_{\mathrm{i}}^{\mathrm{a}(x, q(x)) \boldsymbol{\Phi}_{\mathrm{i}}^{\mathrm{q}}(x) d x} .
$$


L'egalité (5.3) s'obtient alors par intégration par parties.

Pour obtenir des résultats de régularité sur les solutions de l'équation adjointe de (1.1), nous ferons !'hypothèse suivante sur $f$.

$$
f \in C^{1}\left(\Omega \times \mathbb{R}^{m}\right)
$$

(5.4.ii) Il existe des constantes positives $a_{9}, a_{10}, a_{11}$ telles que, pour $x \in \Omega$, tout $u \in \mathbb{R}^{\mathrm{m}}$, on ait:

$$
\begin{aligned}
& |f(x, u)| \leqslant a_{9}|u|^{r}+L(x) \\
& \left|f_{x}(x, u)\right| \leqslant a_{10}|u|^{s}+M(x) \\
& \left|f_{i n}(x, u)\right| \leqslant a_{11}|u|^{s\left(2-p^{\prime}\right)}+N(x),
\end{aligned}
$$

avec $L \in \mathrm{L}^{n p^{\prime} /\left(n-2+p^{\prime}\right)}(\Omega), M \in \mathrm{L}^{p}(\Omega), N \in \mathrm{L}^{p^{*} /\left(2-p^{\prime}\right)}(\Omega), r=\left(n-2+p^{\prime}\right)(p-1) /(n-p)$ et $s=n(p-1) /(n-p)$ si $p<n$ et $r$ et $s$ quelconques dans $[1, \infty[$ si $p \geqslant n$.

Proposition 5.3: Si $p \geqslant 2$ et si les hypothèses (2.4) à (2.7) et (5.4) sont vérifiées, alors les solutions adjointes de (I.I) appartiennent à $W_{i_{0}{ }^{n} p^{*} /\left(n+p^{\prime}-2\right)}\left(\Omega, \mathbb{R}^{n m}\right)$ et les solutions de (I.I) appartiennent $\dot{a}$ $W_{i o c}^{i n g}\left(\Omega, \mathbb{R}^{m}\right)$ si $n>2$ et $\dot{a} W_{\text {loc }}^{\text {r }}$ pour tout $r<+\infty$ si $n=2$.

Preuve: Ce résultat est établi dans la proposition 7.1 de [10] lorsque les fonctions $a_{i}^{\mathrm{a}}$ ne dépendent pas de $x$. Compte tenu des hypothèses faites sur $a_{i}^{\mathrm{a}}$ et des résultats du paragraphe 4 , la démonstration de la proposition 5.3 est analogue à celle de la proposition 7.1 et du théorème 6.3 de [10].

On remarquera toutefois que les conditions de croissance en $u$ imposées à $f$ sont plus restrictives que celles de [10] qui sont suffisantes pour établir le résultat de régularité locale (cf.[10] hypothése 7.5). On démontre dans ce paragraphe le

Théorème 5.4: Si $p \geqslant 2$ et si les hypothèses (2.1), (2.3) à (2.7). (5.4) sont véri-

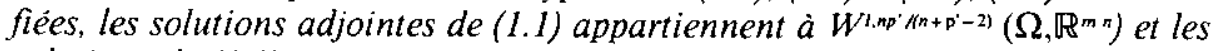
solutions de (l.l) appartiennent $\dot{a} W^{I . n p /(n-2)}\left(\Omega, \mathbb{R}^{m}\right)$ si $n>2$ et $\dot{a} W^{i r r}\left(\Omega, \mathbb{R}^{m}\right)$ pour lout $r<+\infty$ si $n=2$.

Pour démontrer le théorème 5.4, nous adaptons les méthodes développées dans la preuve du théorème 3.2 à l'équation (5.3). Avec les notations du paragraphe 3 , le changement de variable $y=\psi(x)$ effectué dans (5.3) conduit à l'équation:

$$
\int_{\mathbb{R}_{+}^{n}} B_{j}^{a}(y, \tilde{q}(y)) \tilde{\Phi}(y) J^{-1}(y) d y=
$$




$$
=-\int_{\mathbb{R}_{+}^{\mathrm{n}}} \tilde{\Phi}_{\mathrm{j} \beta}^{\mathrm{a}}(y) T_{\mathrm{a}}^{\beta}(y) \tilde{u}^{i}(y) J^{-1}(y) d y .
$$

où

$$
\begin{gathered}
\tilde{q}(y)=q\left(\psi^{-1}(y)\right), \tilde{u}(y)=u\left(\psi^{-1}(y)\right), \\
B_{i}^{q}(y, w)=b_{i}^{a}\left(\psi^{-1}(y), w\right), \\
\tilde{\Phi} q(y)=\Phi_{i}^{\mathrm{a}}\left(\psi^{-1}(y)\right), \tilde{\Phi}_{\mathrm{ip}}^{\mathrm{a}}(y)=\frac{\partial}{\partial y_{\beta}} \tilde{\Phi}_{i}^{\mathrm{a}}(y) .
\end{gathered}
$$

On suppose en outre que, dans $(5.5), \tilde{\Phi}$ appartient à $W_{i o c}^{1}\left(\mathbb{R}_{+}^{n} \cap B(0, R), \mathbb{R}^{\mathrm{mn}}\right)$.

Démontrons tout d'abord le résultat suivant, qui est l'analogue de la proposition 3.3.

Proposition 5.5: Si une solution $\tilde{q}$ de (5.5) appartient $\dot{a} W^{h s}\left(\mathbb{R}_{+}{ }_{+} \cap B(0, r)\right.$, $\mathbb{R}^{\mathrm{m}}$ ) avec $p^{\prime} \leqslant s \leqslant n p^{\prime} /(n-2)$ et $p^{\prime} \leqslant 2$, alors, pour tout $\alpha \in\{1, \ldots, n-1\}$, $\frac{\partial}{\partial y_{\alpha}} \tilde{q}$ appartient $\dot{a} L^{k(s)}\left(\mathbb{R}_{+}^{\mathrm{n}} \cap B(0, r), \mathbb{R}^{\mathrm{mn}}\right)$ avec $k(s)=2 s /\left(s+2-p^{\prime}\right)$, (on pose $n p^{\prime} /(n-2)=+\infty$ si $\left.n=2\right)$.

Preuve de la proposition 5.5: Ici encore, la preuve repose sur la méthode des translations de Nirenberg. De facon à simplifier la démonstration, nous remplacerons formellement comme au paragraphe 3 les quotients différentiels $\Delta_{-h}^{\gamma}$ et $\Delta_{h}^{\gamma}$ par les derivées partielles $-\frac{\partial}{\partial y_{\gamma}}$ et $\frac{\partial}{\partial y_{\gamma}}$. On substitue $-\frac{\partial}{\partial y_{\gamma}}\left(\frac{\partial}{\partial y_{y}} \tilde{q}(.) \xi().\right)$ à $\check{\Phi}$ dans (5.5) (où $\xi$ est definie en (3.9)), on transpose l'opérateur $-\frac{\partial}{\partial y_{\gamma}}$ et on obtient:

$$
\begin{aligned}
& \int_{\mathrm{R}_{+}^{\mathrm{n}}} \frac{\partial}{\partial y_{\gamma}}\left(B_{\mathrm{i}}^{\mathrm{a}}(.) J^{-1}(y)\right) \tilde{q}_{\mathrm{i \gamma}}^{\mathrm{a}}(y) \xi(y) d y \\
= & -\int_{\mathrm{R}_{+}^{\mathrm{n}}} \tilde{q}_{\mathrm{i} \beta \gamma}^{\mathrm{a}}(y) \xi(y) \frac{\partial}{\partial y_{\gamma}}\left(T_{\mathrm{a}}^{\mathrm{a}}(y) \tilde{u}^{\mathrm{i}}(y) J^{-1}(y)\right) d y- \\
- & \int_{\mathrm{R}_{+}^{\mathrm{a}}} \tilde{q}_{\mathrm{i}}^{\mathrm{a}}(y) \xi_{\beta}(y) \frac{\partial}{\partial \mathrm{y}_{\gamma}}\left(T_{\mathrm{a}}^{\mathrm{p}}(y) \tilde{u}^{\mathrm{i}}(y) J^{-1}(y)\right) d y,
\end{aligned}
$$


où

$$
B_{\mathrm{i}}^{\mathrm{a}}(.)=B_{\mathrm{i}}^{\mathrm{a}}(y, \tilde{q}(y)) \text { et } \hat{q}_{\gamma}(y)=\frac{\partial}{\partial y_{\gamma}} \tilde{q}(y) .
$$

On pose:

$$
\begin{aligned}
& B_{\mathrm{i} \gamma}^{\mathrm{u}}(.)=B_{\mathrm{i} \gamma}^{\mathrm{a}}(y, \hat{q}(y)) \text { et } B_{\mathrm{i} j}^{\alpha \gamma}(.)=B_{\mathrm{ij}}^{\mathrm{a} \beta}(y, \tilde{q}(y)) \text {, } \\
& I_{1}=\int_{\mathrm{R}_{+}^{\mathrm{n}}} B_{\mathrm{i} \gamma}^{\mathrm{a}}(.) J^{-1}(y) \tilde{q}_{\mathrm{i} \gamma}^{\mathrm{a}}(y) \xi(y) d y, \\
& I_{2}=\int_{\mathrm{R}_{+}^{\mathrm{n}}} B_{\mathrm{i} j}^{\mathrm{\alpha \beta}}(.) \tilde{q}_{\mathrm{j}}^{\beta}(y) \tilde{q}_{\mathrm{iy}}^{\mathrm{a}}(y) \mathrm{J}^{-1}(y) \xi(p) d y, \\
& I_{3}=\int_{\mathrm{R}_{+}^{\mathrm{n}}} B_{\mathrm{i}}^{\mathrm{o}}(.) J_{\gamma}^{-1}(y) \tilde{q}_{\mathrm{i} \gamma}^{\mathrm{a}}(y) \xi(y) d y, \\
& I_{4}=-\int_{\mathrm{R}_{+}^{\mathrm{n}}} \tilde{q}_{\mathrm{i} y \mathrm{y}}^{\mathrm{a}}(y) \xi(y) T_{\mathrm{a} \gamma}^{\mathrm{B}}(y) \tilde{u}^{i}(y) J^{-1}(y) d y, \\
& I_{s}=-\int_{\mathrm{R}_{+}^{\mathrm{n}}} \tilde{q}_{\mathrm{i \beta \gamma}}^{\mathrm{a}}(y) \xi(y) T_{\mathrm{a}}^{\mathrm{g}}(y) \tilde{u}_{\gamma}^{j}(y) J^{-1}(y) d y, \\
& I_{6}=-\int_{\mathrm{R}_{+}^{\mathrm{a}}} \tilde{q}_{\mathrm{s} \gamma}^{\mathrm{a}}(y) \xi(y) T_{\mathrm{a}}^{\mathrm{a}}(y) \tilde{u}^{i}(y) J_{\mathrm{r}}^{-1}(y) d y, \\
& I_{7}=-\int{\tilde{\mathrm{R}^{\mathrm{n}}}}_{+} \tilde{q}_{\mathrm{\gamma} \gamma}^{\mathrm{a}}(y) \xi_{\beta}(y) T_{\mathrm{\alpha}}^{\mathrm{\beta}}(y) \tilde{u}^{\prime}(y) J^{-1}(y) d y \text {, }
\end{aligned}
$$

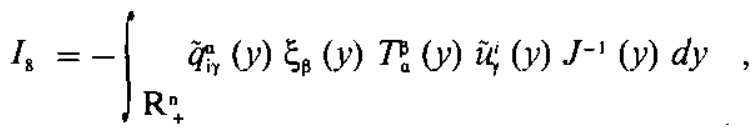

$$
\begin{aligned}
& I_{q}=-\int_{\mathrm{R}_{+}^{\mathrm{n}}} \tilde{q}_{\mathrm{i \gamma}}^{\mathrm{a}}(y) \xi_{\beta}(y) T_{\mathrm{a}}^{\mathrm{p}}(y) \tilde{u}^{i}(y) J_{\gamma}^{-1}(y) d y .
\end{aligned}
$$

Avec ces notations, (5.6) est équivalent à:

$$
I_{1}+I_{2}+I_{3}=I_{4}+I_{5}+I_{6}+I_{7}+I_{3}+I_{9}
$$

Compte tenu de (5.1.ii), on a: .

$$
q_{\mathrm{in}}^{\mathrm{a}}(x)=-f^{i}(x, u(x))=-\tilde{f^{i}}(y, \tilde{u}(y)) .
$$


Avec l'égalité: $q_{\text {ia }}^{\mathrm{a}}(x)=-\tilde{q}_{\mathrm{i}}^{\mathrm{a}}(y) T_{\mathrm{a}}^{\mathrm{B}}(y)$, on obtient:

$$
\left.\tilde{q}_{\mathrm{i} \hat{\gamma}}^{\mathrm{a}}(y) T_{\mathrm{a}}^{\mathrm{\beta}}(y)=-\tilde{\left(f^{i}\right.}(y, \tilde{u}(y))\right)_{\mathrm{r}}-\tilde{q}_{\mathrm{p}}^{\mathrm{a}}(y) T_{\mathrm{\sigma \gamma}}^{\mathrm{\beta}}(y) .
$$

On posera $(\tilde{f},(y, \tilde{u}(y)))_{\gamma}=(\tilde{f},(.))_{\gamma}$. Compte tenu de $(5.8)$; on a:

$$
\begin{aligned}
& I_{4}=\int_{\mathrm{R}_{+}^{\mathrm{p}}}\left(\tilde{f}^{i}\right)_{\gamma} \xi\left(T_{\mathrm{a}}^{\mathrm{p}}\right)^{-1} T_{\mathrm{a} \gamma}^{\mathrm{p}} \tilde{u}^{\mathrm{j}} J^{-1}+\int_{\mathrm{R}_{+}^{\mathrm{a}}} \tilde{q}_{\mathrm{i} \beta}^{\mathrm{a}} \xi T_{\mathrm{a}}^{\mathrm{\beta}}\left(T_{\mathrm{a}}^{\mathrm{p}}\right)^{-2}\left(T_{\mathrm{a} \gamma}^{\mathrm{\beta}}\right)^{2} \tilde{u}^{i} J^{-1} \\
& =\int_{\mathrm{R}_{+}^{\mathrm{a}}}\left(\tilde{f^{\mathrm{i}}}\right)_{\gamma} \xi\left(T_{\mathrm{a}}^{\mathrm{\beta}}\right)^{-1} T_{\mathrm{\alpha} \gamma}^{\beta} \tilde{u}^{\mathrm{i} J} J^{-1}-\int_{\mathrm{R}_{+}^{\mathrm{n}}} \tilde{f^{\mathrm{i}}} \xi\left(T_{\mathrm{a}}^{\mathrm{\beta}}\right)^{-2}\left(T_{\mathrm{a \gamma}}^{\mathrm{\beta}}\right)^{2} \tilde{u}^{i} J^{-1}
\end{aligned}
$$

Par des calculs analogues, on obtient:

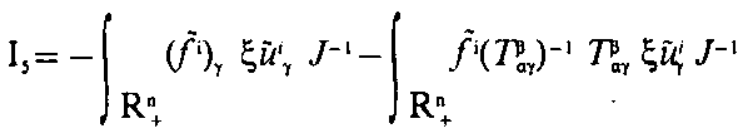

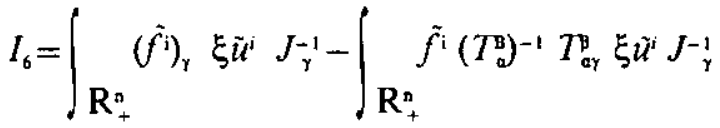

Par un calcul analogue à celui de la minoration de $I_{5}$ au paragraphe 3 et compte tenu de (4.4), on obtient:

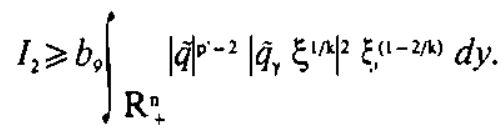

Avec les estimations (4.1) et (4.2), on obtient:

$$
\left|I_{\mathrm{l}}\right|+\left|I_{3}\right| \leqslant c_{\mathrm{l}} \int_{\mathrm{R}_{+}^{\mathrm{n}_{+}}}\left|\tilde{q}^{\mid p^{2}-1}\right| \hat{q}_{y} \xi^{(-1 / \mathrm{k} \mid} \mid \xi^{(1-1 / \mathrm{k})} d y
$$

où $c_{1}$ dépend de $b_{1}, b_{2}$ et $\psi$. De (5.9), (5.10), (5.11), on déduit:

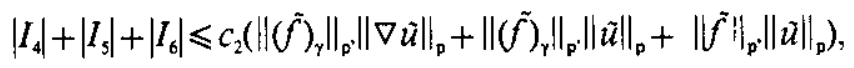

ou $c_{2}$ ne dépend que de $\xi$ et du difféomorphisme $\psi$. Par un calcul direct et avec l'inégalité de Hölder, on obtient:

$$
\begin{aligned}
& \left\|(\tilde{f})_{\gamma}\right\|_{p^{\prime}} \leqslant\left\|\tilde{f_{\gamma_{\gamma}}}(., \tilde{u}(.))\right\|_{p^{\prime}}+\left\|\left|\tilde{f_{u}}(., \tilde{u}(.))\right| \cdot \mid \nabla \tilde{u}\right\|_{p^{\prime}} \\
& \leqslant\left\|\tilde{f}_{\gamma_{\gamma}}(., \tilde{u}(.))\right\|_{p^{\prime}}+\left\|\tilde{f_{v}}(., \tilde{u}(.))\right\|_{\left.p^{\prime} / 2-p^{\prime}\right\}}\|\nabla \tilde{u}\|_{p^{\prime}}
\end{aligned}
$$


Avec (5.14), (5.15) et l'hypothèse 5.4 , on obtient:

$$
\left|I_{4}\right|+\left|I_{5}\right|+\left|I_{6}\right| \leqslant c_{3},
$$

oú $c_{3}$ ne dépend pas de $k$. Compte tenu de (3.9.iv), on a aussi:

$$
\begin{gathered}
\left|I_{7}\right|+\left|I_{8}\right|+\left|I_{9}\right| \leqslant c_{4}\left\{\int_{\mathrm{R}_{+}^{\mathrm{n}}}\left|\tilde{q}^{\xi^{1 / 2 / k}}\right||\tilde{u}| d y+\int_{\mathrm{R}_{+}^{\mathrm{n}}}\left|\tilde{q}_{\gamma} \xi^{1 / k}\right||\nabla u| d y\right\} \\
\leqslant c_{4}\left\{\left\|\tilde{q}_{\gamma} \xi^{1 / k}\right\|_{\mathrm{p}^{\cdot}}\|\tilde{u}\|_{\mathrm{p}}+\left\|\tilde{q}_{Y} \xi^{1 / k}\right\|_{p^{\prime}}\|\nabla \tilde{u}\|_{\mathrm{p}}\right\} .
\end{gathered}
$$

De (5.8), (5.12), (5.13), (5.16), (5.17), on déduit:

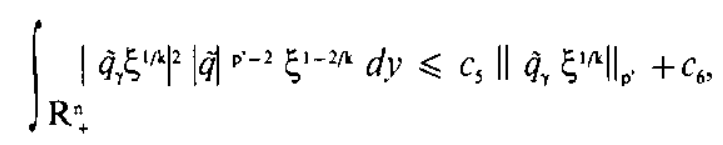

oú $\mathcal{c}_{\mathrm{s}}$ et $\mathcal{C}_{\mathrm{s}}$ dépendent de $\|\nabla u\|_{\mathrm{p}}$ et $\|u\|_{\mathrm{p}}$ mais ne dépendent pas de $k$.

La fin est alors identique à la 3ème étape de la preuve de la proposition 3.3.

Démontrons maintenant un résultat analogue à celui de la proposition 3.4.

Proposition 5.6: Si une solution $\tilde{q}$ de (5.5) appartient à $W^{\prime \prime}\left(\mathbb{R}_{+}^{n} \cap B(0, r), \mathbb{R}^{m n}\right)$ avec $p^{\prime} \leqslant s \leqslant n p^{\prime} /(n-2)$ et $p^{\prime} \leqslant 2$, alors $\dot{q}$ appartient $\dot{a}$ $W^{l, k(s)}\left(\mathbb{R}_{+}^{n} \cap B(0, r), \mathbb{R}^{m n}\right)$, avec $k(s)=2 s /(s+2-p)$.

Preuve de la proposition 5.6. lère étape: On procède comme pour la proposition 3.4. De la proposition 5.3, on déduit que $\tilde{q}$ admet des dérivées partielles au sens classique presque partout dans $\mathbb{R}_{+}^{n} \cap B(0, r)$. On note:

$\hat{B}=\left\{y \in \mathbb{R}^{\mathrm{n}}{ }_{+} \cap B(0, r) \mid \hat{q}\right.$ admet des dérivées partielles premières en $\left.y\right\}$

et $N=\left\{y \in \hat{B} \mid \tilde{q}(y)=0_{\mathrm{mn}}\right\}$.

On démontre alors (cf. lère étape de la preuve de la proposition 3.4) que $\tilde{q}_{y}(y)$ $=0_{\mathrm{mn}}$ presque partout sur $\bar{N}$ et pour tout $\gamma \in\{1, \ldots, n\}$.

2 ème étape: On estime $\tilde{q}_{n}$ sur $\left(\mathbb{R}_{+}^{n} \cap B(0, r)\right\} \backslash \bar{N}$.

Remarquons tout d'abord que $\tilde{q}$ et $\nabla \tilde{u}$ sont presque partout dérivables sur $\left(\mathbb{R}_{+}^{n} \cap B(0, r)\right) \backslash \bar{N}$ et que l'estimation (3.17) est vérifiée en presque tout point de $\left(\mathbb{R}_{+}^{n} \cap B(0, r)\right) \backslash \bar{N}$. En dérivant l'égalité: 


$$
\tilde{u}_{\mathrm{\beta}}^{i}(y)=B(y, \tilde{q}(y)) \mathrm{T}_{\mathrm{B}}^{-1, a}(y)
$$

par rapport à $y_{8}$, on obtient:

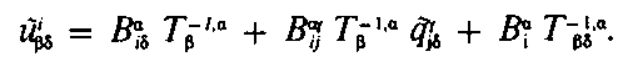

De (5.19) et des estimations (4.1) à (4.4), on déduit l'existence d'une constante $c$, telle que:

$$
\left|\nabla \tilde{u}_{\delta}\right| \leqslant c_{7}\left\{|\tilde{q}|^{p^{\prime}-1}+|\tilde{q}|^{p-2}\left|\tilde{\mathrm{q}}_{\delta}\right|\right\} \text { si } \delta \neq n .
$$

Avec (5.18) on a aussi:

$$
|\nabla \tilde{u}| \leqslant c_{\beta}|\tilde{q}|^{p^{*}-1} \text {. }
$$

De (5.20), (5.21) et (3.17), on déduit:

$$
\left|\tilde{u}_{n m}\right| \leqslant c_{8}\left\{|\tilde{q}|^{p-1}+\sum_{s=n}|\tilde{q}|^{p-1}\left|\tilde{q}_{\delta}\right|+|\tilde{f}(.)||\tilde{q}|^{p^{-2}}\right\}
$$

De (5.19), on déduit également:

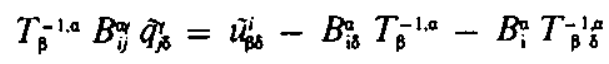

La matrice carré d'ordre $(m+n)$ :

$$
\left(C_{i j}^{q}\right)=\left(T_{i j}^{-1, a} B_{i j}^{\alpha i}\right)
$$

peut s'écrire par blocs de la facon suivante:

$$
\left(\mathrm{C}_{\mathrm{ij}}\right)=\left(\mathrm{T}^{\mathrm{t}} \delta_{\mathrm{ik}}\right)\left(\mathrm{B}_{\mathrm{kj}}\right),
$$

où $\delta_{i k}$ est le symbole de Krönecker. (Le second membre de l'égalité est un produit matriciel par blocs). On en déduit que cette matrice est inversible et que:

$$
\left(C_{i j}\right)^{-1}=\left(A_{k j}\right)\left({ }^{1} T^{\mathrm{s}}{ }_{i k}\right),
$$

soit encore:

$$
\left(C_{\mathrm{ij}}^{\mathrm{a}}\right)^{-1}=\left(A_{\mathrm{ij}}^{\mathrm{a}} T_{\mathrm{a}}^{\mathrm{h}}\right)
$$

En prenant $\delta=n$ dans (5.23) et avec (5.24), on a:

$$
\hat{q}_{\mathrm{in}}^{\mathrm{a}}=A_{i j}^{\mathrm{g}} T_{\beta}^{\mathrm{b}}\left(\tilde{\mathrm{u}}_{\mathrm{in}}^{\mathrm{i}}-B_{\mathrm{in}}^{\mathrm{a}} T_{\beta}^{-1, a}-B_{i}^{\mathrm{a}} T_{\beta_{\mathrm{n}}^{-1, a}}^{-1,}\right) .
$$

De $(5.20),(5.22),(5.25),(4.1)$ à (4.4), on déduit: 


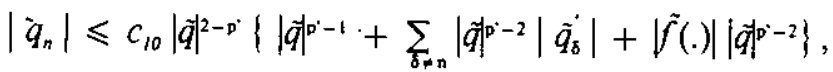

soit encore:

$$
\left.\left|\tilde{q}_{n}\right| \leqslant c_{10}|| \tilde{q}\left|+\sum_{\delta+\infty}\right| \tilde{q}_{\delta}|+| \tilde{f}(.) \mid\right\} .
$$

Avec la proposition 5.5, on a:

$$
\dot{q}_{i 6}^{\mathrm{a}} \in \mathrm{L}^{\mathrm{k}(\mathrm{s})} \text { si } \delta \neq n
$$

L'hypothèse (5.4ii) et l'estimation (5.26) permettent maintenant de montrer que $\dot{q} \in \mathrm{W}^{1, k(s)}\left(\mathbb{R}_{+}^{\mathrm{n}} \cap \mathrm{B}(0, \mathrm{r}), \mathbb{R}^{\mathrm{mn}}\right)$ et on peut obtenir une mejoration de $\|\tilde{q}\|_{\mathbf{w}^{1, k(s)}}$ indépendante de $k(s)$.

Preuve du théorème 5.4: Par un raisonnement identique à celui de la preuve du théorème 3.2 , avec la proposition 5.6 , on démontre que les solutions adjointes de (1.1) appartiennent à $W^{1, n p^{\prime} /\left(n+p^{\prime}-2\right)}\left(\Omega, R^{m n}\right)$.

Avec l'égalité $u_{i}^{i}(x)=b_{i}^{a}(x, q(x))$ et avec (4.1), on obtient le résultat de régularité pour les solutions de (1.1).

\section{COMMENTAIRES ET CONCLUSION}

Dans l'exemple classique où l'on a:

$$
a_{i}^{a}(x, v)=d(x)|v|^{p-2} v_{a}^{i} \text { avec } d \in \mathbf{W}^{1, \infty}(\Omega) \text { et } d(x)>c>0,
$$

les fonctions $a_{i}^{a}$ vérifient les hypothèses (2.3) à (2.7). Nous nous sommes limités ici aux problèmes avec conditions aux limites homogènes. Dans ce cadrelà, les théorèmes 3.2 et 5.4 appliqués au problème associé à (6.1) améliorent les résultats relatifs à ce problème donnés dans [13] et [15].

Dans [13], J. SIMON étudie ce problème avec des conditions aux limites non homogènes et obtient des résultats de régularité dans les espaces de BESOV, mais pour $p$ inférieur à 2, l'ordre de dérivabilité qu'il obtient est strictement inférieur à 2. Le probléme suivant donc ouvert:

Quelles sont les conditions aux limites pour lesquelles les solutions du probléme associé à $(6.1)$ appartiennent à $W^{2, n p /(n+p-2)}\left(\Omega, R^{m}\right)$ quand $p<2$ ?

Pour conclure, il est important de noter que les hypothèses (2.1) à (2.7) pour lesquelles on obtient les résultats de régularité locale et les résultats de régularité globale, dépassent largement le cadre des problémes pour lesquels on ob- 
tient des résultats de continuitế Höldérienne ([8], [17], [18], [19]), c'est ce qui rend les théorèmes 3.2 et 5.4 particulièrement intéressants.

\section{Bibliographie}

[1] R. ADAMS, Sobolev spaces. Academic Press 1975.

[2] J. DenY et J.-L. Lions, Les espaces du type Beppo Levi. Ann. Inst. Fourier vol. 5, 1953-54, pp. 305-370.

[3] E. DiBENEDETTO, $C^{++a}$ local regularity of weak solutions of degenerate elliptic equations. Non Linear Analysis, Theory, Methods and Appl. vol. $7, \mathrm{~N}^{\circ} 8, \mathrm{pp} .827-850,1983$.

[4] E. DIBENEDETTO and A. FRIEDMAN, Regularity of solutions of non linear degenerate parabolic systems. J. für reine u. angew. Math. (1984) 349, pp. 83-128.

[5] L. C. EVANS, $A$ new proof of local $C^{\text {ha }}$ regularity for solutions of certain degenerate elliptic P.D.E. J. Diff. Eq. 45, 1982, pp. 356-375.

[6] I. EKEland et R. TEMAN, Analyse convexe et problèmes variationnels (Dunod 1974).

[7] M. GIAQUINTA, Multiple integrals in the calculus of variations and non linear elliptic systems. Princeton University Press 1983.

[8] M. GIAQUINTA, and G. MODICA, Remarks on the regularity of the minimizers of certain degenerate functionnals. Man. Math. 57 (1986), 55-99.

[9] G. N. JAKOVLEV, Properties of solutions of a class of second order quasilinear elliptic equation in divergence form. Proc. Steklov Inst. Math. 131, 1974, 242-252.

[10] J.-P. RAYMOND, Théorèmes de régularité locale pour des systèmes elliptiques dégénérés et des problèmes non différentiables (à paraitre Ann.Fac. Sc. Toulouse).

[11] S. SAKS, Theory of Integral (New York, Hafner 1937).

[12] J. Simon, Sur des équations aux dérivées partielles non linéaires. Thèse, Paris 1977 (Université P. et M. Curie).

[13] J. SIMON, Regularité de la solution d'un problème aux limites non linéaires. Ann. Fac. Sc. Toulouse, vol. 3, 1981.

[14] G. STAMPACCHIA, Equations elliptiques du second ordre à coeficients discontinus (Montréal: Université de Montréal $\mathrm{N}^{\circ} 16,1986$ ).

[15] F. de THELIN, Régularité de la solution d'un problème de Dirichlet fortement non linéaire. Thèse Toulouse, 1981.

[16] F. de THELIN, Local regularity properties for the solutions of a non linear partial differential equation. Non Linear Analysis T.M.A. Vol. 6, $\mathrm{N}^{\circ} 8$, 1982

[17] P. TOLKSDORF, Regularity for a more general class of Quasilinear Elliptic Equations. J.D.E. 51 (1984), pp. 126-150

[18] P. TOLKSDORF, Everywhere regularity for some quasilinear systems with lack of ellipticity. Ann. Mat.Pura Appl.(4) 134 (1983), 241-266. 
[19] K. UHLENBECK, Regularity for a class of non linear elliptic systems. Acta Math. 138 (1977), pp. 219-240.

[20] D. Gilbarg, N. S. Trudinger, Elliptic Partial Differential Equations of Second Order. Springer (1977).

Laboratoire d'Analyse Numérique

Université Paul Sabatier

1 18, Route de Narbonne

31062 Toulouse Cedex

FRANCE

Recibido: 12 diciembre 1988 